\title{
Diversity-Multiplexing Tradeoff in Selective Cooperation for Cognitive Radio
}

\author{
Yulong Zou, Member, IEEE, Yu-Dong Yao, Fellow, IEEE, and Baoyu Zheng, Senior Member, IEEE
}

\begin{abstract}
In this paper, we first explore a selective cooperation framework for secondary user transmissions in a cognitive radio network with single relay. In the selective cooperation framework, two transmission modes (i.e., relay diversity transmission and non-relay direct transmission) are considered. We study two specific selective cooperation schemes with and without an acknowledgement (ACK) from a cognitive destination as to if it succeeds in decoding or not, called ACK and nonACK based selective cooperation, respectively. We derive closedform outage probability expressions for the two schemes with imperfect spectrum sensing, showing that an outage probability floor occurs in high signal-to-noise ratio (SNR) regions due to mutual interference between primary and secondary users. We consider the use of the outage probability floor to generalize the traditional diversity-multiplexing tradeoff (DMT) definition, based on which a DMT analysis is conducted for the nonACK and ACK based selective cooperation schemes. We then extend the selective cooperation framework to a multiple-relay cognitive radio network considering the best cognitive relay only to participate in assisting secondary transmissions, referred to as the selective best-relay cooperation. We also consider the nonACK and ACK based selective best-relay cooperation schemes and develop their DMTs by using the generalized DMT definition.
\end{abstract}

Index Terms-Cooperative diversity, cognitive radio, diversitymultiplexing tradeoff, selective cooperation, outage probability, diversity gain.

\section{INTRODUCTION}

C OGNITIVE radio [1], [2] is proposed as a means to enable secondary users to communicate with each other over primary user's licensed bands through detecting spectrum holes. Typically, a secondary user first searches an available spectrum hole through spectrum sensing and then transmits its data traffic over the detected hole [3]-[5]. However, it is impossible to achieve perfect spectrum sensing without any miss detection and false alarm of the presence of primary users, implying that mutual interference between the primary and secondary users exists in cognitive radio networks. Since

Paper approved by F. Santucci, the Editor for Wireless System Performance of the IEEE Communications Society. Manuscript received March 29, 2011; revised February 16, 2012.

This work was partially supported by the Postgraduate Innovation Program of Scientific Research of Jiangsu Province (Grant Nos. CX08B_080Z, CX09B_150Z), the Key Project of Nature Science Funding of Jiangsu Province (GrantNo. BK2010077), and the National Natural Science Foundation of China (Grant No. 60972039).

Y. Zou is with the Institute of Signal Processing and Transmission, Nanjing University of Posts and Telecommunications, Nanjing, Jiangsu 210003, China. He is also with the Electrical and Computer Engineering Department, Stevens Institute of Technology, Hoboken, NJ 07030 USA (e-mail: yulong.zou@gmail.com).

Y.-D. Yao is with the Electrical and Computer Engineering Department, Stevens Institute of Technology, Hoboken, NJ 07030, USA (e-mail: yyao@stevens.edu).

B. Zheng is with the Institute of Signal Processing and Transmission, Nanjing University of Posts and Telecommunications, Nanjing, Jiangsu 210003, China (e-mail: zby@njupt.edu.cn).

Digital Object Identifier 10.1109/TCOMM.2012.072612.110180 a primary user's quality-of-service (QoS) requirement shall be guaranteed in cognitive radio [3], the mutual interference would severely degrade the secondary user transmission performance.

Cooperative diversity, emerging as a spatial diversity technique, has been proposed to improve the wireless transmission performance. In [6], [7], several cooperative protocols, i.e., fixed relaying, selection relaying, and increment relaying, have been proposed and analyzed for Rayleigh fading channels in terms of the outage probability. The advantages of such cooperative diversity protocols [6]-[8] typically come at the cost of a reduction in spectral efficiency since the cooperative relays transmit over multiple orthogonal channels to avoid interfering with each other. To overcome this shortcoming, the relay selection based cooperative diversity has been studied in [9] and [10], where only the "best" relay is selected to participate in relaying and thus only two channels (i.e., the best relay link and direct link) are required regardless of the number of relays. It has been shown that the best-relay selection based cooperative diversity protocol not only avoids complex synchronization among distributed relays, but also achieves the full diversity [9].

The cooperation technique also has great potential to be applied in cognitive radio networks. In [11], a collaborative spectrum sensing approach has been proposed to combat wireless fading and improve the probability of detection of the primary user. In [12], the authors have explored the application of cooperative diversity to spectrum sensing by exploiting the user cooperation. Then, paper [13] has studied a fully distributed cooperative spectrum sensing strategy in which cognitive users are allowed to share their sensing information. Furthermore, in [14], the authors have explored coalition games for distributed collaborative spectrum sensing and shown the performance improvement of proposed coalition formation algorithm. Note that, the cooperative spectrum sensing approaches proposed in [11]-[14] require dedicated reporting channels for cognitive users to transmit their binary detection results or observed signals to a fusion center. To remove the dedicated reporting channels, we have proposed an alternative cooperative spectrum sensing framework in [16] and shown that the proposed scheme (without dedicated reporting channels) can achieve the same sensing performance as conventional cooperation spectrum sensing (with dedicated reporting channels). In [17], we have considered the use of cooperative diversity for secondary transmissions with a primary QoS constraint and proposed an adaptive cooperation diversity scheme with best-relay selection in cognitive radio networks considering a spectrum underlay sharing policy.

In this paper, we explore a selective cooperation framework for cognitive radio by jointly considering the relay diversity transmission and non-relay direct transmission. Typically, the 


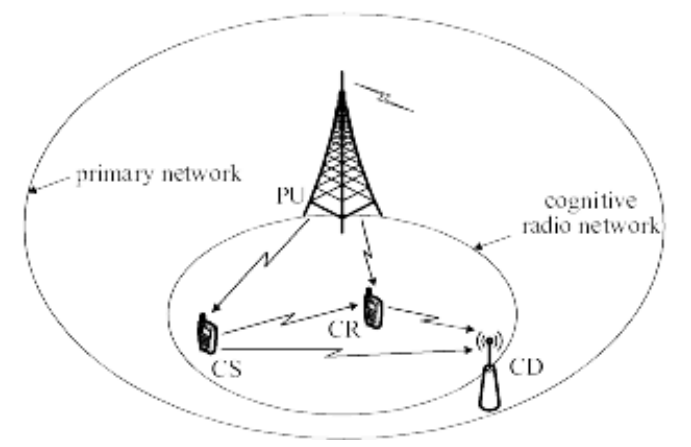

Fig. 1. Coexistence of a cognitive radio network and a primary network.

relay transmission achieves diversity gain at the cost of loss of multiplexing gain and, in contrast, the other mode inherits full multiplexing but without any diversity gain. We study the non-ACK and ACK based selective cooperation schemes for secondary transmissions and derive their closed-form outage probability expressions over Rayleigh fading channels. We generalize the traditional definitions of diversity gain and multiplexing gain and develop DMTs of the non-ACK and ACK based selective cooperation schemes. We extend the selective cooperation to a multiple-relay cognitive radio network and study a selective best-relay cooperation scenario where only the best cognitive relay is selected to participate in assisting secondary transmissions. We also consider the nonACK and ACK based selective best-relay cooperation schemes and develop DMTs of the non-ACK and ACK based selective best-relay cooperation schemes.

The remainder of this paper is organized as follows. In Section II, we provide a brief description of the selective cooperation framework in a cognitive radio network and present signal modeling for the non-ACK and ACK based selective cooperation schemes. Section III derives closedform outage probability expressions for the two schemes over Rayleigh fading channels, followed by Section IV, where a DMT analysis is presented. Next, in Section V, we extend the selective cooperation to a multiple-relay cognitive radio network and propose the non-ACK and ACK based selective best-relay cooperation schemes. In this section, we derive closed-form outage probability expressions and conduct DMT analysis for the non-ACK and ACK based selective best-relay cooperation schemes. Finally, in Section VI, we make some concluding remarks.

\section{Proposed Non-ACK And ACK based Selective COOPERATION SCHEMES IN COgnitive Radio NETWORKS}

This section presents a non-ACK and an ACK based selective cooperation transmission schemes in cognitive radio networks. We formulate the signal modeling for the two selective cooperation schemes.

\section{A. System Model}

Consider that a cognitive radio network coexists with a primary network, as shown in Fig. 1, where the cognitive radio network consists of a cognitive source (CS), a cognitive relay $(\mathrm{CR})$, and a cognitive destination $(\mathrm{CD})$. As stated in [3], [4], CS shall first sense the licensed frequency band

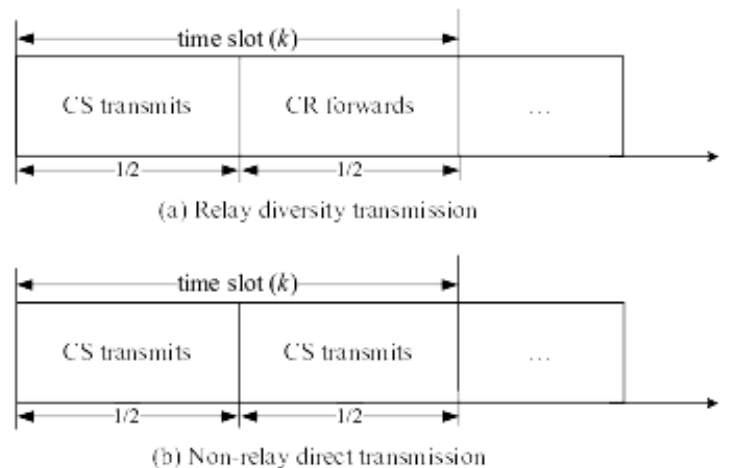

Fig. 2. Illustration of the proposed selective cooperation framework: (a) relay diversity transmission, and (b) non-relay direct transmission.

in search of spectrum holes and then utilize the detected holes for data transmissions. If CS misses a detection of the presence of $\mathrm{PU}$, the secondary transmissions would interfere with primary transmissions. In order to show the impact of spectrum sensing on the secondary transmission performance, we consider imperfect spectrum sensing with the probabilities of detection and false alarm of the presence of primary user (PU) as denoted by $P_{d}$ and $P_{f}$, respectively. According to IEEE 802.22 requirements, the detection and false alarm probabilities should be guaranteed to target values, i.e., $P_{d} \geq 0.9$ and $P_{f} \leq 0.1$. Hence, throughout this paper, we consider $P_{d}=0.99$ and $P_{f}=0.01$ to protect a primary QoS.

Once a spectrum hole was detected, CS would start its data transmissions through the detected hole. Following [5] and [6], we consider the use of cognitive relays for CS' data transmissions and study a selective cooperation framework as shown in Fig. 2, where two transmission modes (i.e., relay diversity transmission and non-relay direct transmission) are available and CS would adaptively select a preferable choice depending on a specific strategy. Typically, the relay transmission mode can achieve the full diversity gain at the cost of loss of multiplexing gain and, in contrast, the direct transmission mode can achieve the full multiplexing gain. This motivates us to explore the selective cooperation by jointly considering the two transmission modes for potential performance improvement. Note that, although the cognitive radio scenario in this paper considers a single channel, the proposed protocols can be applied and extended to a more generic scenario with multiple channels through wideband spectrum sensing. Once more than one spectrum holes were found, the cognitive source starts its data transmissions over the detected spectrum holes by using multi-carrier transmission techniques (e.g., orthogonal frequency-division multiplexing), for which similar performance results can be obtained.

For notational convenience, let $H_{p}(k)$ denote whether or not the licensed band is occupied by $\mathrm{PU}$ in time slot $k$, i.e., $H_{p}(k)=H_{0}$ represents that the band is unoccupied by PU; otherwise, $H_{p}(k)=H_{1}$. We use a Bernoulli distribution with parameter $P_{a}$ to model the random variable $H_{p}(k)$, i.e., $\operatorname{Pr}\left[H_{p}(k)=H_{0}\right]=P_{a}$ and $\operatorname{Pr}\left[H_{p}(k)=H_{1}\right]=1-P_{a}$. Let $\hat{H}_{s}(k)$ denote the decision (made at CS) on the status of a licensed band, e.g., $\hat{H}_{s}(k)=H_{0}$ represents that an idle licensed band is detected at CS. Note that the spectrum sensing result $\hat{H}_{s}(k)$ is assumed to be unchanged during one time slot and varies slot-by-slot. Assume that all the channels are 
modeled as independent complex Gaussian random variables and are invariant within each time slot. All receivers have the additive white Gaussian noise (AWGN) with the same power spectral density $N_{0}$.

\section{B. Direct Transmission}

For the comparison purpose, let us first consider the direct data transmission from CS to $\mathrm{CD}$ without relaying. Given that a spectrum hole was detected (i.e., $H_{s}(k)=H_{0}$ ), CS would transmit its signal $x_{s}(k)\left(E\left(\left|x_{s}(k)\right|^{2}\right)=1\right)$ with power $P_{s}$ at data rate $R$. Denoting the transmit power of PU by $P_{p}$, we can calculate an instantaneous mutual information in time slot $k$ from $\mathrm{CS}$ and $\mathrm{CD}$ via direct transmission as

$$
I_{\text {direct }}(k)=\log _{2}\left(1+\frac{\left|h_{s d}(k)\right|^{2} \gamma_{s}}{\left|h_{p d}(k)\right|^{2} \gamma_{p}|\theta(k)|^{2}+1}\right)
$$

where $\gamma_{s}=P_{s} / N_{0}, \gamma_{p}=P_{p} / N_{0}, h_{s d}(k)$ and $h_{p d}(k)$ are, respectively, the fading coefficients of the channel from CS to $\mathrm{CD}$ and that from PU to $\mathrm{CD}$, and $\theta(k)$ is defined as

$$
\theta(k)= \begin{cases}x_{p}(k), & H_{p}(k)=H_{1} \\ 0, & H_{p}(k)=H_{0}\end{cases}
$$

where $x_{p}(k)$ is the transmit signal at PU in time slot $k$. Note that $H_{p}(k)=H_{0}$ represents that the licensed channel is unoccupied by PU and nothing is transmitted from PU, and $H_{p}(k)=H_{1}$ represents that a primary signal $x_{p}(k)$ $\left(E\left(\left|x_{p}(k)\right|^{2}\right)=1\right)$ is transmitted.

\section{Non-ACK Based Selective Cooperation}

In what follows, we study a non-ACK based selective cooperation scheme, where no acknowledgement (with regard to success or not in decoding) would be transmitted from CD. No matter whether $\mathrm{CD}$ decodes the received signal directly from CS successfully or not, CR would always forward its decoded outcome if it succeeds in decoding CS' signal. Specifically, in the first phase, CS broadcasts its data to CR and CD. Then, in the subsequent phase, if $\mathrm{CR}$ decodes the received information successfully, it would forward the decoded result to CD that would finally combine the signal copies received during the two phases. Otherwise, CS, instead of CR, transmits new data information which is different from the one transmitted during the previous phase. We can employ a cyclic redundancy code (CRC) to determine whether $\mathrm{CR}$ decodes its received signal successfully or not, i.e., if the CRC checking passes, it is assumed that $\mathrm{CR}$ succeeds in decoding.

In the given case $\hat{H}_{s}(k)=H_{0}$, CS would broadcast its signal $x_{s}(k)$ to $\mathrm{CR}$ and $\mathrm{CD}$ in the first phase of time slot $k$. Thus, the instantaneous mutual information from CS to CR, $I_{s r}(k)$, can be given by

$$
I_{s r}(k)=\frac{1}{2} \log _{2}\left(1+\frac{\left|h_{s r}(k)\right|^{2} \gamma_{s}}{\left|h_{p r}(k)\right|^{2} \gamma_{p}|\theta(k, 1)|^{2}+1}\right)
$$

where the factor $1 / 2$ in the front of $\log$-function is resulted from a half-duplex relaying constraint [5], [6], $h_{s r}(k)$ and $h_{p r}(k)$ are, respectively, the fading coefficients of the channel from CS to CR and that from PU to CR, and $\theta(k, 1)$ is defined as

$$
\theta(k, 1)= \begin{cases}x_{p}(k, 1), & H_{p}(k)=H_{1} \\ 0, & H_{p}(k)=H_{0}\end{cases}
$$

where $x_{p}(k, 1)$ is the PU's signal in the first phase of time slot $k$. During the second phase of time slot $k$, CR would forward its decoded outcome using a selective relaying strategy. As shown in Fig. 2, there are two possible cases depending on whether CR succeeds in decoding CS' signal or not. In an information-theoretic sense [5], [6], we can describe the event of successfully decoding at $\mathrm{CR}$ as $I_{s r}(k)>R$, where $R$ is the data rate of secondary transmissions. In this case, $C R$ would forward its decoded outcome to the $\mathrm{CD}$ during the second phase of time slot $k$. Combining the received signals during the two phases with maximum ratio combining (MRC), we can obtain an enhanced signal version at $\mathrm{CD}$, and thus the mutual information from CS to $\mathrm{CD}$ using the relay diversity transmission can be given by

$$
\begin{aligned}
I_{\text {relay }}(k)=\frac{1}{2} \log _{2}(1 & +\frac{\left|h_{s d}(k)\right|^{2} \gamma_{s}}{\left|h_{p d}(k)\right|^{2} \gamma_{p}|\theta(k, 1)|^{2}+1} \\
& \left.+\frac{\left|h_{r d}(k)\right|^{2} \gamma_{s}}{\left|h_{p d}(k)\right|^{2} \gamma_{p}|\theta(k, 2)|^{2}+1}\right)
\end{aligned}
$$

where $\theta(k, 2)$ is defined as

$$
\theta(k, 2)= \begin{cases}x_{p}(k, 2), & H_{p}(k)=H_{1} \\ 0, & H_{p}(k)=H_{0}\end{cases}
$$

wherein $x_{p}(k, 2)$ is the PU's signal in the second phase of time slot $k$. Given the case that CR fails to decode CS' signal (i.e., $I_{s r}(k)<R$ ), CS (instead of CR) would transmit new data information. The reason for choosing the direct transmission mode in this case is that CR would not help CD in decoding CS' signal, if it forwards an incorrectly decoded CS' signal to $\mathrm{CD}$. Thus, in this case, the mutual information from CS to CD can be depicted by (1).

\section{ACK Based Selective Cooperation}

In this subsection, we consider an ACK based selective cooperation scheme, where $\mathrm{CD}$ is assumed to transmit an acknowledgement to inform CS and CR whether it decodes CS' signal successfully or not in the first phase. Specifically, if $\mathrm{CD}$ succeeds in decoding its received signal directly from CS in the first phase, it broadcasts an ACK signal to both CS and $\mathrm{CR}$ so that $\mathrm{CR}$ can avoid forwarding the decoded outcome and save the channel resource for CS transmitting new data information. Otherwise, in the case of CD failing to decode CS' signal in the first phase, the process in the subsequent phase of the ACK based selective cooperation scheme is the same as that in the non-ACK based scheme. Throughout this paper, we assume that the power consumption and date rate of the ACK packet are negligible compared to these of an information packet, since the ACK packet typically contains only 1-bit information in an information-theoretical sense.

Accordingly, for the ACK based selective cooperation scheme, the relay diversity transmission mode would be adopted only when $\mathrm{CD}$ fails to decode and CR succeeds in decoding CS' signal in the first phase, i.e., $I_{\text {direct }}(k)<R$ and $I_{s r}(k)>R$. In addition, in the case of $I_{s r}(k)<R$ (implying that CR fails to decode CS' signal), the non-relay direct transmission mode would be employed. 


\section{Outage Analysis of the Proposed Selective COOPERATION SCHEMES}

In this section, we analyze the outage probability of the proposed selective cooperation schemes over Rayleigh fading channels. In the following, let us first consider the direct transmission.

\section{A. Direct Transmission}

As known in [5] and [6], if the mutual information falls below a predefined data rate, an outage event is said to occur. Hence, given that a spectrum hole was detected, an outage probability of the direct transmission can be calculated as

$$
\text { Pout }_{\text {direct }}=\operatorname{Pr}\left[I_{\text {direct }}(k)<R \mid \hat{H}_{s}(k)=H_{0}\right]
$$

where $I_{\text {direct }}(k)$ is given by (1). Using the total probability theorem and conditional probabilities, we can expand the preceding equation as

$$
\begin{aligned}
\text { Pout }_{\text {direct }}= & \operatorname{Pr}\left[I_{\text {direct }}(k)<R \mid H_{p}(k)=H_{0}, \hat{H}_{s}(k)=H_{0}\right] \\
& \times \operatorname{Pr}\left[H_{p}(k)=H_{0} \mid \hat{H}_{s}(k)=H_{0}\right] \\
+ & \operatorname{Pr}\left[I_{\text {direct }}(k)<R \mid H_{p}(k)=H_{1}, \hat{H}_{s}(k)=H_{0}\right] \\
& \times \operatorname{Pr}\left[H_{p}(k)=H_{1} \mid \hat{H}_{s}(k)=H_{0}\right]
\end{aligned}
$$

By using Bayes' Theorem, term $\operatorname{Pr}\left[H_{p}(k)=H_{0} \mid \hat{H}_{s}(k)=\right.$ $H_{0}$ ] as given in (8) can be further calculated as

$$
\begin{aligned}
& \operatorname{Pr}\left[H_{p}(k)=H_{0} \mid \hat{H}_{s}(k)=H_{0}\right] \\
& =\frac{\operatorname{Pr}\left[\hat{H}_{s}(k)=H_{0} \mid H_{p}(k)=H_{0}\right] \operatorname{Pr}\left[H_{p}(k)=H_{0}\right]}{\sum_{i=0,1} \operatorname{Pr}\left[\hat{H}_{s}(k)=H_{0} \mid H_{p}(k)=H_{i}\right] \operatorname{Pr}\left[H_{p}(k)=H_{i}\right]} \\
& =\frac{P_{a}\left(1-P_{f}\right)}{P_{a}\left(1-P_{f}\right)+\left(1-P_{a}\right)\left(1-P_{d}\right)}
\end{aligned}
$$

where $P_{a}=\operatorname{Pr}\left[H_{p}(k)=H_{0}\right]$ is the probability of the licensed band being unoccupied by $\mathrm{PU}, P_{d}=\operatorname{Pr}\left[\hat{H}_{s}(k)=\right.$ $\left.H_{1} \mid H_{p}(k)=H_{1}\right]$ and $P_{f}=\operatorname{Pr}\left[\hat{H}_{s}(k)=H_{1} \mid H_{p}(k)=\right.$ $\left.H_{0}\right]$ are, respectively, the probabilities of detection and false alarm of the presence of PU. For notional convenience, let $\pi_{1}=P_{a}\left(1-P_{f}\right) /\left[P_{a}\left(1-P_{f}\right)+\left(1-P_{a}\right)\left(1-P_{d}\right)\right]$ and $\pi_{2}=\left(1-P_{a}\right)\left(1-P_{d}\right) /\left[P_{a}\left(1-P_{f}\right)+\left(1-P_{a}\right)\left(1-P_{d}\right)\right]$, respectively. Substituting $I_{\text {direct }}(k)$ from (1) into (8) yields

$$
\begin{aligned}
\text { Pout }_{\text {direct }}= & \pi_{1} \operatorname{Pr}\left[\left|h_{s d}(k)\right|^{2}<\Delta\right] \\
& +\pi_{2} \operatorname{Pr}\left[\left|h_{s d}(k)\right|^{2}-\left|h_{p d}(k)\right|^{2} \gamma_{p} \Delta<\Delta\right]
\end{aligned}
$$

where $\Delta=\left(2^{R}-1\right) / \gamma_{s}$. One can observe from the expressions of $\pi_{1}$ and $\pi_{2}$ that, with either an increased detection probability or decreased false probability, $\pi_{1}$ increases and $\pi_{2}$ decreases, which result in the decrease of outage probability as shown in (9), since $\operatorname{Pr}\left[\left|h_{s d}(k)\right|^{2}<\Delta\right]$ is always less than $\operatorname{Pr}\left[\left|h_{s d}(k)\right|^{2}-\left|h_{p d}(k)\right|^{2} \gamma_{p} \Delta<\Delta\right]$. Notice that both the random variables $\left|h_{s d}(k)\right|^{2}$ and $\left|h_{p d}(k)\right|^{2}$ follow exponential distributions with means $\sigma_{s d}^{2}$ and $\sigma_{p d}^{2}$, respectively. Therefore, the probabilities $\operatorname{Pr}\left[\left|h_{s d}(k)\right|^{2}<\Delta\right]$ and $\operatorname{Pr}\left[\left|h_{s d}(k)\right|^{2}-\left|h_{p d}(k)\right|^{2} \Delta \gamma_{p}<\Delta\right]$ are given by $1-\exp \left(-\frac{\Delta}{\sigma_{s d}^{2}}\right)$ and $1-\frac{\sigma_{s d}^{2}}{\sigma_{p d}^{2} \gamma_{p} \Delta+\sigma_{s d}^{2}} \exp \left(-\frac{\Delta}{\sigma_{s d}^{2}}\right)$, respectively. Without loss of generality, the primary transmit SNR $\gamma_{p}$ can be represented by a product of the secondary transmit SNR $\gamma_{s}$ and a coefficient $\alpha$, i.e., $\gamma_{p}=\alpha \gamma_{s}$. Following (9) and considering $\gamma_{s} \rightarrow \infty$, we can obtain

$$
\text { Pout }_{\text {direct,floor }}=\frac{\alpha \pi_{2}}{\alpha\left(2^{R}-1\right) \lambda_{s d-p d}^{-1}+1} \cdot \frac{2^{R}-1}{\lambda_{s d-p d}}
$$

where $\lambda_{s d-p d}=\sigma_{s d}^{2} / \sigma_{p d}^{2}$ is the ratio of the channel gain from $\mathrm{CS}$ to $\mathrm{CD}$ to the channel gain from PU to $\mathrm{CD}$. As indicated from (10), an outage probability floor occurs as the transmit SNRs, $\gamma_{s}$ and $\gamma_{p}$, approach infinity. The reason is that, when $\gamma_{s}$ and $\gamma_{p}$ are sufficiently high, the interference from the primary user becomes dominant factor to cause an outage event in secondary channels. Hence, in high SNR regions, it is not feasible to improve the outage performance by increasing the transmit power, which motivates us to explore approaches to reduce the outage probability floor.

\section{B. Non-ACK Based Selective Cooperation}

In this subsection, let us focus on the outage analysis for the proposed non-ACK based selective cooperation scheme. As mentioned in Section II-C, given a spectrum hole detected, the relay diversity transmission mode would be utilized only if CR succeeds in decoding CS' signal (i.e., $I_{s r}(k)>R$ ); Otherwise, the non-relay direct transmission mode would be adopted by $\mathrm{CS}$ for its data transmissions. Hence, given the case $\hat{H}_{s}(k)=H_{0}$, an outage probability of the non-ACK selective cooperation scheme is given by

$$
\begin{aligned}
\text { Pout }_{\text {non-ACK }}= & \operatorname{Pr}\left[I_{\text {relay }}(k)<R, I_{s r}(k)>R \mid \hat{H}_{s}(k)=H_{0}\right] \\
& +\operatorname{Pr}\left[I_{\text {direct }}(k)<R, I_{s r}(k)<R \mid \hat{H}_{s}(k)=H_{0}\right]
\end{aligned}
$$

which can be rewritten as Eq. (12) at the top of the following page, where $\pi_{1}=P_{a}\left(1-P_{f}\right) /\left[P_{a}\left(1-P_{f}\right)+\left(1-P_{a}\right)\left(1-P_{d}\right)\right]$ and $\pi_{2}=\left(1-P_{a}\right)\left(1-P_{d}\right) /\left[P_{a}\left(1-P_{f}\right)+\left(1-P_{a}\right)\left(1-P_{d}\right)\right]$. Hence, substituting (1), (3) and (5) into (12) yields

$$
\begin{aligned}
\text { Pout }_{\text {non-ACK }}= & \pi_{1} \operatorname{Pr}\left[\left|h_{s d}(k)\right|^{2}+\left|h_{r d}(k)\right|^{2}<\Lambda\right] \operatorname{Pr}\left[\left|h_{s r}(k)\right|^{2}>\Lambda\right] \\
+ & \pi_{2} \operatorname{Pr}\left[\left|h_{s d}(k)\right|^{2}+\left|h_{r d}(k)\right|^{2}-\left|h_{p d}(k)\right|^{2} \gamma_{p} \Lambda<\Lambda\right] \\
& \times \operatorname{Pr}\left[\left|h_{s r}(k)\right|^{2}-\left|h_{p r}(k)\right|^{2} \gamma_{p} \Lambda>\Lambda\right] \\
+ & \pi_{1} \operatorname{Pr}\left[\left|h_{s d}(k)\right|^{2}<\Delta\right] \operatorname{Pr}\left[\left|h_{s r}(k)\right|^{2}<\Lambda\right] \\
+ & \pi_{2} \operatorname{Pr}\left[\left|h_{s d}(k)\right|^{2}-\left|h_{p d}(k)\right|^{2} \gamma_{p} \Delta<\Delta\right] \\
& \times \operatorname{Pr}\left[\left|h_{s r}(k)\right|^{2}-\left|h_{p r}(k)\right|^{2} \gamma_{p} \Lambda<\Lambda\right]
\end{aligned}
$$

wherein $\Lambda=\left(2^{2 R}-1\right) / \gamma_{s}$. Notice that random variables $\left|h_{s d}(k)\right|^{2},\left|h_{s r}(k)\right|^{2},\left|h_{p d}(k)\right|^{2},\left|h_{p r}(k)\right|^{2}$ and $\left|h_{r d}(k)\right|^{2}$ follow exponential distributions with means $\sigma_{s d}^{2}, \sigma_{s r}^{2}, \sigma_{p d}^{2}, \sigma_{p r}^{2}$ and $\sigma_{r d}^{2}$, respectively. Thus, the probabilities as given in (13), e.g., $\operatorname{Pr}\left[\left|h_{s r}(k)\right|^{2}>\Lambda\right], \operatorname{Pr}\left[\left|h_{s r}(k)\right|^{2}-\left|h_{p r}(k)\right|^{2} \gamma_{p} \Lambda>\Lambda\right]$, $\operatorname{Pr}\left[\left|h_{s d}(k)\right|^{2}<\Delta\right], \operatorname{Pr}\left[\left|h_{s r}(k)\right|^{2}<\Lambda\right]$, and $\operatorname{Pr}\left[\left|h_{s d}(k)\right|^{2}-\right.$ $\left.\left|h_{p d}(k)\right|^{2} \gamma_{p} \Delta<\Delta\right]$, can be easily found in closed-form solutions, where $\operatorname{Pr}\left[\left|h_{s r}(k)\right|^{2}>\Lambda\right]$ and $\operatorname{Pr}\left[\left|h_{s r}(k)\right|^{2}-\right.$ $\left.\left|h_{p r}(k)\right|^{2} \gamma_{p} \Lambda>\Lambda\right]$ are calculated as $\exp \left(-\frac{\Lambda}{\sigma_{s r}^{2}}\right)$ and $\frac{\sigma_{s r}^{2}}{\sigma_{p r}^{2} \gamma_{p} \Lambda+\sigma_{s r}^{2}} \exp \left(-\frac{\Lambda}{\sigma_{s r}^{2}}\right)$, respectively. In addition, denoting $x=\left|h_{r d}(k)\right|^{2}, y \stackrel{s r}{=}\left|h_{s d}(k)\right|^{2}$ and $z=\left|h_{p d}(k)\right|^{2}$, we can rewrite $\operatorname{Pr}\left[\left|h_{s d}(k)\right|^{2}+\left|h_{r d}(k)\right|^{2}<\Lambda\right]$ and $\operatorname{Pr}\left[\left|h_{s d}(k)\right|^{2}+\right.$ $\left.\left|h_{r d}(k)\right|^{2}-\left|h_{p d}(k)\right|^{2} \gamma_{p} \Lambda<\Lambda\right]$ as $\operatorname{Pr}[x+y<\Lambda]$ and 


$$
\begin{aligned}
\text { Pout }_{\text {non-ACK }}= & \pi_{1} \operatorname{Pr}\left[I_{\text {relay }}(k)<R, I_{s r}(k)>R \mid \hat{H}_{s}(k)=H_{0}, H_{p}(k)=H_{0}\right] \\
& +\pi_{2} \operatorname{Pr}\left[I_{\text {relay }}(k)<R, I_{s r}(k)>R \mid \hat{H}_{s}(k)=H_{0}, H_{p}(k)=H_{1}\right] \\
& +\pi_{1} \operatorname{Pr}\left[I_{\text {direct }}(k)<R, I_{s r}(k)<R \mid \hat{H}_{s}(k)=H_{0}, H_{p}(k)=H_{0}\right] \\
& +\pi_{2} \operatorname{Pr}\left[I_{\text {direct }}(k)<R, I_{s r}(k)<R \mid \hat{H}_{s}(k)=H_{0}, H_{p}(k)=H_{1}\right]
\end{aligned}
$$

$\operatorname{Pr}\left[x+y-z \gamma_{p} \Lambda<\Lambda\right]$, which are calculated as

$$
\operatorname{Pr}[x+y<\Lambda]= \begin{cases}1-\left(1+\frac{\Lambda}{\sigma_{s d}^{2}}\right) \exp \left(-\frac{\Lambda}{\sigma_{s d}^{2}}\right), & \sigma_{s d}^{2}=\sigma_{r d}^{2} \\ 1-\frac{\sigma_{s d}^{2}}{\sigma_{s d}^{2}-\sigma_{r d}^{2}} \exp \left(-\frac{\Lambda}{\sigma_{s d}^{2}}\right) & \text { otherwise } \\ -\frac{\sigma_{r d}^{2}}{\sigma_{r d}^{2}-\sigma_{s d}^{2}} \exp \left(-\frac{\Lambda}{\sigma_{r d}^{2}}\right) & \end{cases}
$$

and

$$
\operatorname{Pr}\left[x+y-z \gamma_{p} \Lambda<\Lambda\right]=\operatorname{Pr}[x+y<\Lambda]+\vartheta
$$

where a closed-form solution to $\operatorname{Pr}[x+y<\Lambda]$ is given in (14), and the parameter $\vartheta$ is given by

$$
\vartheta= \begin{cases}\frac{\mathrm{H}_{s d}}{\sigma_{s d}^{2}}\left(\frac{\mathrm{H}_{s d}}{\sigma_{s d}^{2}}+\frac{\Lambda}{\sigma_{s d}^{2}}\right) \exp \left(-\frac{\Lambda}{\sigma_{s d}^{2}}\right), & \sigma_{s d}^{2}=\sigma_{r d}^{2} \\ \frac{\mathrm{H}_{s d}}{\sigma_{s d}^{2}-\sigma_{r d}^{2}} \exp \left(-\frac{\Lambda}{\sigma_{s d}^{2}}\right) & \text { otherwise } \\ +\frac{\mathrm{H}_{r d}}{\sigma_{r d}^{2}-\sigma_{s d}^{2}} \exp \left(-\frac{\Lambda}{\sigma_{r d}^{2}}\right) & \end{cases}
$$

wherein $\mathrm{H}_{s d}=\sigma_{s d}^{2} \sigma_{p d}^{2} \gamma_{p} \Lambda /\left(\sigma_{p d}^{2} \gamma_{p} \Lambda+\sigma_{s d}^{2}\right)$ and $\mathrm{H}_{r d}=$ $\sigma_{r d}^{2} \sigma_{p d}^{2} \gamma_{p} \Lambda /\left(\sigma_{p d}^{2} \gamma_{p} \Lambda+\sigma_{r d}^{2}\right)$. Using Lemma 1 in Appendix A, we can obtain an outage probability floor of the non-ACK based selective cooperation scheme as

$$
\begin{aligned}
\text { Pout }_{\text {non-ACK, floor }}= & \frac{\pi_{2} \alpha^{2}}{\left(\rho^{-1} \lambda_{s d-p d}^{-1} v+1\right)\left(\lambda_{s d-p d}^{-1} v+1\right) \delta} \\
& \times\left(\frac{2^{2 R}-1}{\lambda_{s d-p d}}\right)^{2} \\
& +\frac{\pi_{2} \alpha^{2}}{\left(\lambda_{s d-p d}^{-1} \nu+1\right)\left(\lambda_{s d-p d}^{-1} v+\rho\right)} \\
& \times \frac{\left(2^{2 R}-1\right)\left(2^{R}-1\right)}{\lambda_{s d-p d}^{2}}
\end{aligned}
$$

where $v=\alpha\left(2^{2 R}-1\right), \nu=\alpha\left(2^{R}-1\right), \rho=\lambda_{s r-p r} / \lambda_{s d-p d}$, $\delta=\lambda_{r d-p d} / \lambda_{s d-p d}$, and $\lambda_{s r-p r}=\sigma_{s r}^{2} / \sigma_{p r}^{2}$ is the ratio of the channel gain from CS to $\mathrm{CR}$ to that from PU to $\mathrm{CR}$, and $\lambda_{r d-p d}=\sigma_{r d}^{2} / \sigma_{p d}^{2}$ is the ratio of the channel gain from CR to $\mathrm{CD}$ to that from PU to $\mathrm{CD}$.

\section{ACK Based Selective Cooperation}

The following presents an outage probability analysis for the ACK based selective cooperation scheme. As stated in Section II-D, for the ACK based selective cooperation scheme, the relay diversity transmission mode would be adopted in the case of $I_{\text {direct }}(k)<R$ and $I_{s r}(k)>R$ and, moreover, the non-relay direct transmission mode should be used if $I_{s r}(k)<R$ occurs. Thus, the outage probability of the ACK based selective cooperation scheme can be calculated as Eq. (17) at the top of the following page. Considering the total probability theorem and using (3), (6) and (10), we can rewrite the preceding equation as Eq. (18). All the probabilities as given in the preceding equation can be calculated with closed-form solutions. Notice that $\operatorname{Pr}\left[\left|h_{s d}(k)\right|^{2}+\left|h_{r d}(k)\right|^{2}<\Lambda,\left|h_{s d}(k)\right|^{2}<\Delta\right]$ and $\operatorname{Pr}\left[\left|h_{s d}(k)\right|^{2}+\left|h_{r d}(k)\right|^{2}-\left|h_{p d}(k)\right|^{2} \gamma_{p} \Lambda<\Lambda,\left|h_{s d}(k)\right|^{2}-\right.$ $\left.\left|h_{p d}(k)\right|^{2} \gamma_{p} \Delta<\Delta\right]$ are rewritten as $\operatorname{Pr}[x<\Lambda-y, 0<\Delta-y]$ and $\operatorname{Pr}\left[x<\Lambda-y+z \gamma_{p} \Lambda, 0<\Delta-y+z \gamma_{p} \Delta\right]$, where $x=\left|h_{r d}(k)\right|^{2}, y=\left|h_{s d}(k)\right|^{2}$ and $z=\left|h_{p d}(k)\right|^{2}$, which are calculated in closed-form as

$$
\begin{aligned}
& \operatorname{Pr}[x<\Lambda-y, 0<y] \\
& =\left\{\begin{array}{rr}
1-\exp \left(-\frac{\Delta}{\sigma_{s d}^{2}}\right)-\frac{\Delta}{\sigma_{s d}^{2}} \exp \left(-\frac{\Lambda}{\sigma_{s d}^{2}}\right), & \sigma_{s d}^{2}=\sigma_{r d}^{2} \\
1-\exp \left(-\frac{\Delta}{\sigma_{s d}^{2}}\right)-\frac{\sigma_{r d}^{2}}{\sigma_{r d}^{2}-\sigma_{s d}^{2}} \exp \left(-\frac{\Lambda}{\sigma_{r d}^{2}}\right) & \\
\times\left[1-\exp \left(-\frac{\Delta}{\sigma_{s d}^{2}}+\frac{\Delta}{\sigma_{r d}^{2}}\right)\right] & \text { otherwise }
\end{array}\right.
\end{aligned}
$$

and

$$
\begin{aligned}
& \operatorname{Pr}\left[x<\Lambda-y+z \gamma_{p} \Lambda, 0<\Delta-y+z \gamma_{p} \Delta\right] \\
& = \begin{cases}1 & -\frac{\sigma_{s d}^{2}}{\sigma_{s d}^{2}+\sigma_{p d}^{2} \gamma_{p} \Delta} \exp \left(-\frac{\Delta}{\sigma_{s d}^{2}}\right) \\
- & \frac{\Delta}{\sigma_{s d}^{2}+\sigma_{p d}^{2} \gamma_{p} \Lambda}\left(1+\frac{\sigma_{p d}^{2} \sigma_{s d}^{2} \gamma_{p}}{\sigma_{s d}^{2}+\sigma_{p d}^{2} \gamma_{p} \Lambda}\right), \quad \sigma_{s d}^{2}=\sigma_{r d}^{2} \\
& \times \exp \left(-\frac{\Lambda}{\sigma_{s d}^{2}}\right) \\
1 & -\frac{\sigma_{s d}^{2}}{\sigma_{s d}^{2}+\sigma_{p d}^{2} \gamma_{p} \Delta} \exp \left(-\frac{\Delta}{\sigma_{s d}^{2}}\right)-\xi, \quad \text { otherwise }\end{cases}
\end{aligned}
$$

wherein $\xi=\frac{\sigma_{r d}^{2}}{\sigma_{r d}^{2}+\sigma_{p d}^{2} \gamma_{p} \Lambda} \exp \left(-\frac{\Lambda}{\sigma_{r d}^{2}}\right)\left[\frac{\sigma_{r d}^{2}}{\sigma_{r d}^{2}-\sigma_{s d}^{2}}+\right.$ $\left(\frac{\sigma_{p d}^{2} \gamma_{p} \Delta \sigma_{r d}^{4}}{\sigma_{r d}^{2} \sigma_{p d}^{2} \gamma_{p} \Delta+\sigma_{r d}^{2} \sigma_{s d}^{2}+\sigma_{s d}^{2} \sigma_{p d}^{2} \gamma_{p}(\Lambda-\Delta)}\right.$ $\left.\left.\frac{\sigma_{r d}^{2}}{\sigma_{r d}^{2}-\sigma_{s d}^{2}}\right) \exp \left(-\frac{\Delta}{\sigma_{s d}^{2}}+\frac{\Delta}{\sigma_{r d}^{2}}\right)\right]$. In addition, using Lemma 2 in Appendix A, we can obtain an outage probability floor of the ACK based selective cooperation scheme as

$$
\begin{aligned}
\text { Pout }_{\mathrm{ACK}, \text { floor }}= & \frac{\pi_{2} \alpha^{2}}{\left(\lambda_{s d-p d}^{-1} \nu+1\right)^{2}\left(\rho^{-1} \lambda_{s d-p d}^{-1} v+1\right) \delta} \\
& \times \frac{\left(2^{R}-1\right)\left(2^{2 R}-2^{R}\right)}{\lambda_{s d-p d}^{2}} \\
& +\frac{\pi_{2} \alpha^{2}}{\left(\lambda_{s d-p d}^{-1} \nu+1\right)\left(\rho^{-1} \lambda_{s d-p d}^{-1} v+1\right) \delta} \\
& \times \frac{\left(2^{R}-1\right)\left(2^{2 R}-1\right)}{\lambda_{s d-p d}^{2}} \\
+ & \frac{\pi_{2} \alpha^{2}}{\left(\lambda_{s d-p d}^{-1} \nu+1\right)\left(\lambda_{s d-p d}^{-1} v+\rho\right)} \\
& \times \frac{\left(2^{R}-1\right)\left(2^{2 R}-1\right)}{\lambda_{s d-p d}^{2}} \\
&
\end{aligned}
$$




$$
\begin{aligned}
\operatorname{Pout}_{\mathrm{ACK}}=\operatorname{Pr}\left[I_{\text {relay }}(k)<\right. & \left.R, I_{\text {direct }}(k)<R, I_{s r}(k)>R \mid \hat{H}_{s}(k)=H_{0}\right]+\operatorname{Pr}\left[I_{\text {direct }}(k)<R, I_{s r}(k)<R \mid \hat{H}_{s}(k)=H_{0}\right] \\
\operatorname{Pout}_{\mathrm{ACK}}= & \pi_{1} \operatorname{Pr}\left[\left|h_{s d}(k)\right|^{2}+\left|h_{r d}(k)\right|^{2}<\Lambda,\left|h_{s d}(k)\right|^{2}<\Delta\right] \operatorname{Pr}\left[\left|h_{s r}(k)\right|^{2}>\Lambda\right] \\
& +\pi_{2} \operatorname{Pr}\left[\left|h_{s d}(k)\right|^{2}+\left|h_{r d}(k)\right|^{2}-\left|h_{p d}(k)\right|^{2} \gamma_{p} \Lambda<\Lambda,\left|h_{s d}(k)\right|^{2}-\left|h_{p d}(k)\right|^{2} \gamma_{p} \Delta<\Delta\right] \\
& \times \operatorname{Pr}\left[\left|h_{s r}(k)\right|^{2}-\left|h_{p r}(k)\right|^{2} \gamma_{p} \Lambda>\Lambda\right] \\
& +\pi_{1} \operatorname{Pr}\left[\left|h_{s d}(k)\right|^{2}<\Delta\right] \operatorname{Pr}\left[\left|h_{s r}(k)\right|^{2}<\Lambda\right] \\
& +\pi_{2} \operatorname{Pr}\left[\left|h_{s d}(k)\right|^{2}-\left|h_{p d}(k)\right|^{2} \gamma_{p} \Delta<\Delta\right] \operatorname{Pr}\left[\left|h_{s r}(k)\right|^{2}-\left|h_{p r}(k)\right|^{2} \gamma_{p} \Lambda<\Lambda\right]
\end{aligned}
$$

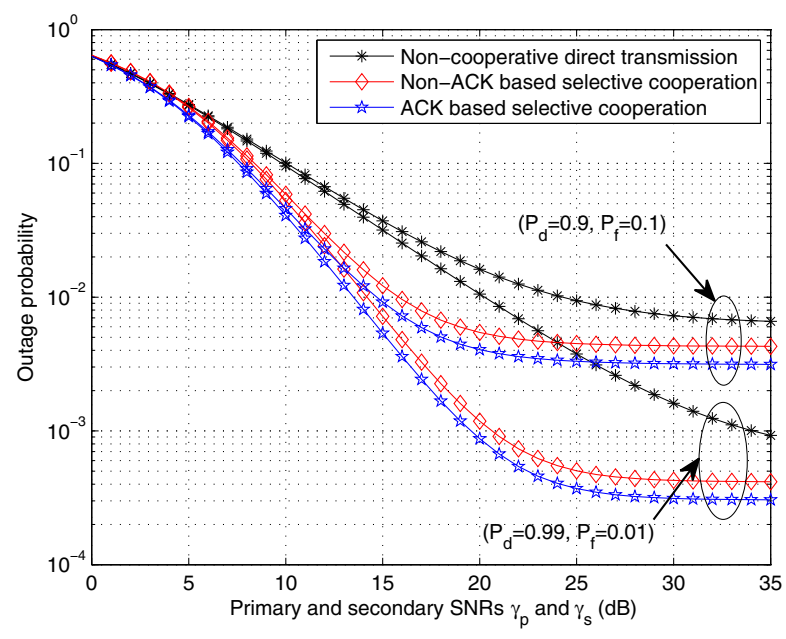

Fig. 3. Outage probability versus primary and secondary SNRs $\gamma_{p}$ and $\gamma_{s}$ of the non-cooperative transmission and selective cooperation schemes with $P_{a}=0.6, R=1 \mathrm{bit} / \mathrm{s} / \mathrm{Hz}, \sigma_{p r}^{2}=\sigma_{p d}^{2}=0.1$, and $\sigma_{s r}^{2}=\sigma_{r d}^{2}=\sigma_{s d}^{2}=1$.

where $\rho=\lambda_{s r-p r} / \lambda_{s d-p d}$ and $\delta=\lambda_{r d-p d} / \lambda_{s d-p d}$.

\section{Numerical Results}

Fig. 3 plots (9), (13) and (18) as a function of the primary and secondary SNRs $\left(\gamma_{p}\right.$ and $\left.\gamma_{s}\right)$ considering two cases, i.e., $\left(P_{d}=0.9, P_{f}=0.1\right)$ and $\left(P_{d}=0.99, P_{f}=0.01\right)$. As depicted in Fig. 3, no matter which transmission scheme is considered, the outage probability corresponding to $\left(P_{d}=\right.$ $\left.0.99, P_{f}=0.01\right)$ is lower that corresponding to $\left(P_{d}=\right.$ $\left.0.9, P_{f}=0.1\right)$, showing the outage probability improvement through better spectrum sensing. All cases in Fig. 3 show that outage probability floors occur in high SNR regions for the non-cooperative direct transmission and the non-ACK and ACK based selective cooperation schemes. This is because that imperfect spectrum sensing results in miss detection of the presence of the primary user, and thus causes mutual interference between the primary and secondary users, which becomes a dominant factor for outage event occurrence in high SNR regions. In addition, one can see from Fig. 3 the ACK based selective cooperation scheme always outperforms the non-ACK based scheme in terms of the outage probability across the whole SNR regions. Note that, although the ACK based selective cooperation scheme achieves better outage performance than that of the non-ACK based scheme, it comes at the expense of delay and is thus only appropriate for nonreal-time data communication applications. In contrast, the non-ACK based selective cooperation scheme is more suitable for real-time applications than the ACK based scheme.

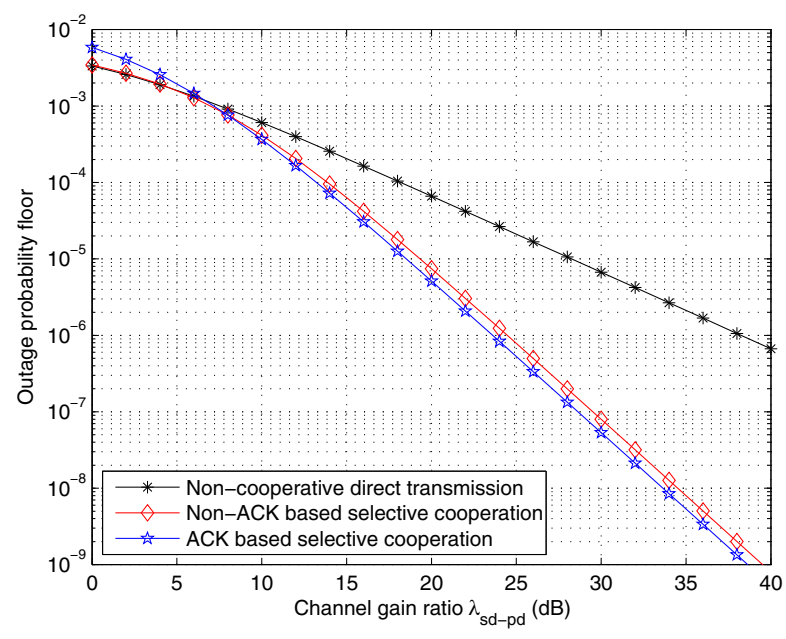

Fig. 4. Outage probability floor versus the channel gain ratio $\lambda_{s d-p d}$ of the non-cooperative transmission and the selective cooperation schemes with $P_{a}=0.6, P_{d}=0.99, P_{f}=0.01, \alpha=1, R=1 \mathrm{bit} / \mathrm{s} / \mathrm{Hz}$, and $\rho=\delta=1$, where $\rho$ and $\delta$ are given by $\rho=\lambda_{s r-p r} / \lambda_{s d-p d}$ and $\delta=\lambda_{r d-p d} / \lambda_{s d-p d}$, respectively.

In Fig. 4, we compare the outage probability floors of the non-cooperative direct transmission and the non-ACK and ACK based selective cooperation schemes by plotting (10), (16), and (21) as a function of the channel gain ratio $\lambda_{s d-p d}$. Note that, if the cognitive radio system employs an advanced signal processing technique (e.g., beam-forming), the interference from a PU transmitter to a SU receiver can be reduced significantly, resulting in a large value of the channel gain ratio, $\lambda_{s d-p d}=\sigma_{s d}^{2} / \sigma_{p d}^{2}$. As shown in Fig. 4, both the non-ACK and ACK based selective cooperation schemes outperform the non-cooperative direct transmission in terms of the outage probability floor performance over wide $\lambda_{s d-p d}$ regions. One can see from Fig. 4 that, in high $\lambda_{s d-p d}$ regions, the slopes of the curves corresponding to the non-ACK and ACK based selective cooperation schemes are twice of that corresponding to the direct transmission. This indicates that, as the channel gain ratio $\lambda_{s d-p d}$ increases, the outage floors of the selective cooperation schemes are reduced much faster than that of the traditional direct transmission.

\section{Diversity-Multiplexing Tradeoff Analysis}

In this section, we investigate the DMT of proposed selective cooperation schemes in cognitive radio networks. As is known in [18], the traditional diversity gain definition is given by $d=-\lim _{\mathrm{SNR} \rightarrow \infty} \log P_{e}(\mathrm{SNR}) / \log$ SNR. If such a definition was used in the diversity analysis of cognitive transmissions, 
the diversity gain would always be zero, since the outage probability of cognitive transmissions approaches a non-zero constant in high SNR regions, as shown in (10), (16) and (21). In order to evaluate the diversity order of cognitive transmissions, we present a generalized diversity gain in [17] as an asymptotic ratio of the outage probability floor to the average gain of an interference link, where the desired link quality is not taken into account. Since both the desired link and interference link come together in cognitive radio due to imperfect spectrum sensing, we here define a diversity gain as an asymptotic ratio of the outage probability floor to a channel gain ratio $\lambda_{s d-p d}$ of the average gains of desired link and interference link as $\lambda_{s d-p d} \rightarrow \infty$. Hence, following (10), the generalized diversity gain of the direct transmission scheme can be given by

$$
d_{\text {direct }}=-\lim _{\lambda_{s d-p d} \rightarrow \infty} \frac{\log \left(\text { Pout }_{\text {direct,floor }}\right)}{\log \left(\lambda_{\text {sd-pd }}\right)}
$$

As discussed in [17], if an advanced signal processing technique (e.g., beam forming) is employed by the cognitive radio system, the interference from a PU transmitter to a SU receiver can be mitigated significantly. Therefore, the channel gain ratio, $\lambda_{s d-p d}$, could approach infinity, when the cognitive users utilize a beam forming technique. In [18], the traditional multiplexing gain $(r)$ is given by $r=\lim _{\mathrm{SNR} \rightarrow \infty} R(\mathrm{SNR}) / \log (\mathrm{SNR})$. Similarly, we here consider the use of $\lambda_{s d-p d}^{\infty}$ (instead of SNR) for a multiplexing gain definition that is analogously given by

$$
r=\lim _{\lambda_{s d-p d} \rightarrow \infty} \frac{R\left(\lambda_{s d-p d}\right)}{\log \left(\lambda_{s d-p d}\right)}
$$

which is applied to the DMT analysis of all protocols including the direct transmission, and the non-ACK and ACK based selection cooperation schemes. Combining (10), (22) and (23) yields

$$
d_{\text {direct }}+r=1
$$

One can observe from (24) that a diversity gain $d_{\text {direct }}=1$ is achieved as $r \rightarrow 0$ and, on the other hand, a full multiplexing gain $r=1$ is achieved as the diversity gain approaches zero. Next, let us examine DMTs of the proposed non-ACK and ACK based selective cooperation schemes. Similar to (22), the diversity gain of the non-ACK based selective cooperation scheme is calculated as

$$
d_{\text {non-ACK }}=-\lim _{\lambda_{s d-p d} \rightarrow \infty} \frac{\log \left(\text { Pout }_{\text {non-ACK,floor }}\right)}{\log \left(\lambda_{s d-p d}\right)}
$$

By combining (16), (23) and (25), a DMT of the non-ACK based selective cooperation scheme can be found as

$$
d_{\text {non-ACK }}+4 r=2
$$

which shows that a diversity gain $d_{\text {non-ACK }}=2$ is achieved as $r \rightarrow 0$ and, however, only one-half multiplexing gain, i.e., $r=1 / 2$, can be achieved as $d_{\text {non-ACK }} \rightarrow 0$. Similarly, the diversity gain of the ACK based selective cooperation scheme is given by

$$
d_{\mathrm{ACK}}=-\lim _{\lambda_{s d-p d} \rightarrow \infty} \frac{\log \left(\text { Pout }_{\mathrm{ACK}, \text { floor }}\right)}{\log \left(\lambda_{\text {sd-pd }}\right)}
$$

Substituting (21) and (23) into (27), we can obtain a DMT of the ACK based selective cooperation scheme as

$$
d_{\mathrm{ACK}}+3 r=2
$$

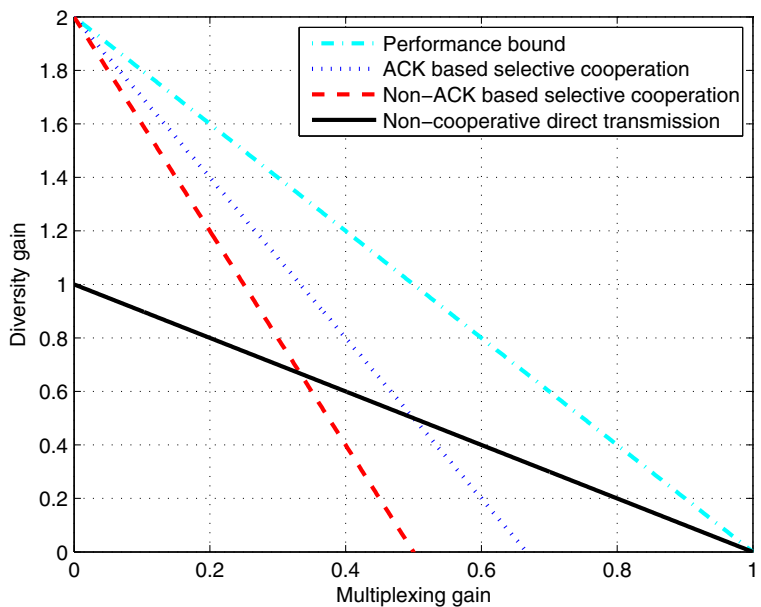

Fig. 5. DMTs for the direct transmission and proposed non-ACK and ACK based selective cooperation schemes.

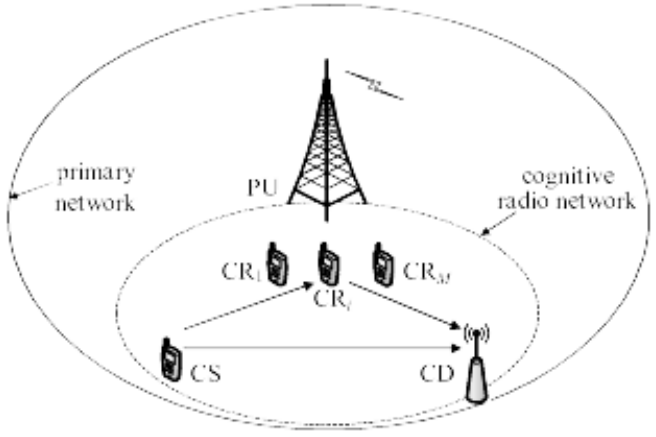

Fig. 6. System model of a multiple-relay cognitive radio network.

One can observe from (28) that a maximum multiplexing gain $r=2 / 3$ is achieved as $d_{\mathrm{ACK}} \rightarrow 0$, showing its advantage over the non-ACK based selective cooperation scheme. Fig. 5 shows a comparison of the DMTs for the direct transmission and the proposed non-ACK and ACK based selective cooperation schemes, where an ideal DMT is also given. It is observed from Fig. 5 that crossover points between the direct transmission and proposed non-ACK and ACK based selective cooperation occur at $r=1 / 3$ and $1 / 2$, respectively. This implies that, if the multiplexing gain is above one-third, i.e., $r>1 / 3$, the non-ACK based selective cooperation is worse than the direct transmission. Moreover, given $r>1 / 2$, the direct transmission even performs better than the ACK based selective cooperation scheme. In addition, the ACK based selective cooperation always outperforms the non-ACK based scheme in terms of the DMT performance.

\section{Extension to Multiple-Relay Cognitive Radio NETWORKS}

In this section, we consider an extension of the selective cooperation to a multiple-relay cognitive radio network consisting of one cognitive source (CS), one cognitive destination (CD), and $M$ cognitive relays (CRs), as shown in Fig. 6. Given multiple CRs available to assist CS' data transmissions, a straightforward way is to allow all the relays to participate in forwarding CS' signal to CD. Although this approach can achieve full diversity gain, it typically requires the complex 

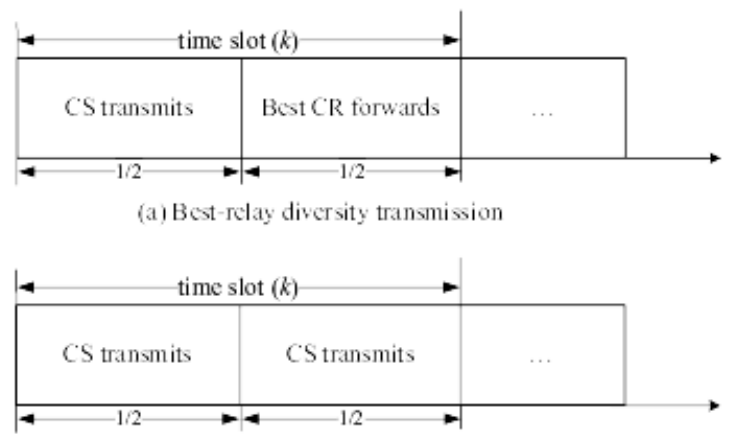

(b) Non-relay direct transmission

Fig. 7. Illustration of the selective best-relay cooperation framework: (a) Best-relay diversity transmission; (b) Non-relay direct transmission.

symbol-level synchronization among distributed relays. To address this issue, an alternative protocol, called the best relay selection, has been studied in cognitive radio networks [17]. It has been shown that the best relay selection also achieves the full diversity for cognitive radio. Hence, we study the selective cooperation in the multiple-relay scenario by considering the best relay only to assist CS' transmissions, called selective best-relay cooperation, as illustrated in Fig. 7 where two transmission modes (i.e., the best-relay diversity transmission and non-relay direct transmission) are considered. One can observe from Fig. 7 that, in the first phase, CS broadcast its data to CD and CRs. Then, all CRs attempt to decode their received signals and those CRs which decode successfully constitute a set $D$, called a decoding set. Accordingly, the sample space of all possible decoding sets can be described as $D \in\left\{\emptyset \cup D_{m}, m=1,2, \cdots 2^{M}-1\right\}$, where $\emptyset$ is an empty set and $D_{m}$ is a non-empty sub-collection of the $M$ cognitive relays. In the subsequent phase, if the decoding set $(D)$ is not empty, the best-relay transmission mode is adopted, i.e., the best relay would be chosen within the decoding set to forward its decoded result to $\mathrm{CD}$. If $D$ is empty, i.e., no relay is able to decode CS' signal, CS would transmit a new data message to CD through its direct link, meaning that the non-relay direct transmission mode is used. For the selective best-relay cooperation, we also consider two strategies: non-ACK and ACK based selective best-relay cooperation. Notice that the only difference between the nonACK and ACK based selective best-relay cooperation is when the best-relay transmission mode should be adopted. To be specific, for the non-ACK based scheme, once the decoding set is not empty, the best-relay transmission mode is employed. However, ACK based selective best-relay cooperation would adopt such transmission mode only when both the decoding set is not empty and CD acknowledges its failure of decoding CS' signal through the direct link.

In what follows, we first present a signal model of the best-relay transmission mode, and then derive closed-form outage probability expressions for the non-ACK and ACK based selective best-relay cooperation schemes. Finally, we investigate the DMT for the proposed selective best-relay cooperation schemes.

\section{A. Signal Model}

In this subsection, we address the signal modeling for the best-relay diversity transmission. Note that a detailed signal model of the direct transmission mode has been provided in Section II-B. Given that a spectrum hole was detected in time slot $k$, i.e., $\hat{H}_{s}(k)=H_{0}$, CS broadcast its message $x_{s}(k)$ with power $P_{s}$ and data rate $R$ to $\mathrm{CD}$ and all CRs. Hence, a mutual information from $\mathrm{CS}$ to $\mathrm{CR}_{i}$, as denoted by $I_{s i}(k)$, is found as

$$
I_{s i}(k)=\frac{1}{2} \log _{2}\left(1+\frac{\left|h_{s i}(k)\right|^{2} \gamma_{s}}{\left|h_{p i}(k)\right|^{2} \gamma_{p}|\theta(k, 1)|^{2}+1}\right)
$$

where $\theta(k, 1)$ is given by $(4), h_{s i}(k)$ and $h_{p i}(k)$ are the fading coefficients of the channel from CS to $\mathrm{CR}_{i}$ and that from $\mathrm{PU}$ to $\mathrm{CR}_{i}$, respectively. We can describe whether $\mathrm{CR}_{i}$ is in decoding $D$ or not as

$$
\begin{array}{ll}
I_{s i}(k)>R, & i \in D_{m} \\
I_{s i}(k)<R, & i \in \bar{D}_{m}
\end{array}
$$

where $\bar{D}_{m}=R-D_{m}$ is the complementary set of $D_{m}$. When the best-relay transmission mode is adopted, we would choose the best cognitive relay within $D_{m}$ to forward its decoded signal to $\mathrm{CD}$. In general, the cognitive relay, which can achieve the highest received signal-to-interference-andnoise ratio (SINR) at $\mathrm{CD}$, is viewed as the "best" relay. Therefore, given that the best-relay transmission mode is used, the mutual information from $\mathrm{CS}$ to $\mathrm{CD}$ via the best cognitive relay through the MRC can be determined by

$$
\begin{aligned}
I_{\text {best-relay }}(k)=\frac{1}{2} \log _{2}( & \frac{\left|h_{s d}(k)\right|^{2} \gamma_{s}}{\left|h_{p d}(k)\right|^{2} \gamma_{p}|\theta(k, 1)|^{2}+1} \\
& \left.+\max _{i \in D_{m}} \frac{\left|h_{i d}(k)\right|^{2} \gamma_{s}}{\left|h_{p d}(k)\right|^{2} \gamma_{p}|\theta(k, 2)|^{2}+1}\right)
\end{aligned}
$$

Now, we complete the model formulation for the best-relay transmission mode.

\section{B. Outage Probability Analysis}

The following focuses on the closed-form outage probability analysis for the non-ACK and ACK based selective best-relay cooperation schemes over Rayleigh fading channels.

1) Non-ACK Based Selective Best-Relay Cooperation: As mentioned above, if the decoding set $D$ is not empty (i.e., $D=D_{m}$ ), the non-ACK based scheme would adopt the bestrelay transmission mode. Otherwise, the direct transmission mode would be used. Hence, an outage probability of the nonACK based selective best-relay cooperation scheme can be calculated as

$$
\begin{aligned}
& \text { Pout }_{\text {non-ACK, multiple }} \\
& =\sum_{m=1}^{2^{M}-1} \operatorname{Pr}\left[I_{\text {best-relay }}(k)<R, D=D_{m} \mid \hat{H}_{s}(k)=H_{0}\right] \\
& \quad+\operatorname{Pr}\left[I_{\text {direct }}(k)<R, D=\emptyset \mid \hat{H}_{s}(k)=H_{0}\right]
\end{aligned}
$$

where $I_{\text {best-relay }}(k)$ and $I_{\text {direct }}(k)$ are given by (31) and (1), respectively. Following (30) and (31), the term $\operatorname{Pr}\left[I_{\text {best-relay }}(k)<\right.$ $\left.R, D=D_{m} \mid \hat{H}_{s}(k)=H_{0}\right]$ at the right-hand side of (32) can be expanded as Eq. (33) at the top of the following page, where $\pi_{1}=P_{a}\left(1-\mathrm{P}_{\mathrm{f}}\right) /\left[P_{a}\left(1-\mathrm{P}_{\mathrm{f}}\right)+\left(1-P_{a}\right)\left(1-P_{d}\right)\right], \pi_{2}=(1-$ $\left.P_{a}\right)\left(1-P_{d}\right) /\left[P_{a}\left(1-\mathrm{P}_{\mathrm{f}}\right)+\left(1-P_{a}\right)\left(1-P_{d}\right)\right]$, and the probabilities $\operatorname{Pr}\left[\left|h_{s i}(k)\right|^{2}>\Lambda\right]$ and $\operatorname{Pr}\left[\left|h_{s i}(k)\right|^{2}-\left|h_{p i}(k)\right|^{2} \gamma_{p} \Lambda>\Lambda\right]$ 


$$
\begin{aligned}
& \operatorname{Pr}\left[I_{\text {best-relay }}(k)<R, D=D_{m} \mid \hat{H}_{s}(k)=H_{0}\right] \\
& =\pi_{1} \operatorname{Pr}\left[\max _{i \in D_{m}}\left|h_{i d}(k)\right|^{2}<\Lambda-\left|h_{s d}(k)\right|^{2}\right] \prod_{i \in D_{m}} \operatorname{Pr}\left[\left|h_{s i}(k)\right|^{2}>\Lambda\right] \prod_{j \in \bar{D}_{m}} \operatorname{Pr}\left[\left|h_{s j}(k)\right|^{2}<\Lambda\right] \\
& +\pi_{2} \operatorname{Pr}\left[\max _{i \in D_{m}}\left|h_{i d}(k)\right|^{2}<\Lambda-\left|h_{s d}(k)\right|^{2}+\left|h_{p d}(k)\right|^{2} \gamma_{p} \Lambda\right] \\
& \quad \times \prod_{i \in D_{m}} \operatorname{Pr}\left[\left|h_{s i}(k)\right|^{2}-\left|h_{p i}(k)\right|^{2} \gamma_{p} \Lambda>\Lambda\right] \prod_{j \in \bar{D}_{m}} \operatorname{Pr}\left[\left|h_{s j}(k)\right|^{2}-\left|h_{p j}(k)\right|^{2} \gamma_{p} \Lambda<\Lambda\right]
\end{aligned}
$$

are given by $\exp \left(-\frac{\Lambda}{\sigma_{s i}^{2}}\right)$ and $\frac{\sigma_{s i}^{2}}{\sigma_{p i}^{2} \gamma_{p} \Lambda+\sigma_{s i}^{2}} \exp \left(-\frac{\Lambda}{\sigma_{s i}^{2}}\right)$, respectively. Denoting $x_{i}=\left|h_{i d}(k)\right|^{2}, y=\left|h_{s d}(k)\right|^{2 i}$ and $z=$ $\left|h_{p d}(k)\right|^{2}$, we can rewrite $\operatorname{Pr}\left[\max _{i \in D_{m}}\left|h_{i d}(k)\right|^{2}<\Lambda-\left|h_{s d}(k)\right|^{2}\right]$ and $\operatorname{Pr}\left[\max _{i \in D_{m}}\left|h_{i d}(k)\right|^{2}<\Lambda-\left|h_{s d}(k)\right|^{2}+\left|h_{p d}(k)\right|^{2} \gamma_{p} \Lambda\right]$ as $\operatorname{Pr}\left[\max _{i \in D_{m}} x_{i}<\Lambda-y\right]$ and $\operatorname{Pr}\left[\max _{i \in D_{m}} x_{i}<\Lambda-y+z \gamma_{p} \Lambda\right]$. Using Eq. (27) of [5], we can obtain a closed-form solution to term $\operatorname{Pr}\left[\max _{i \in D_{m}} x_{i}<\Lambda-y\right]$ as

$$
\begin{aligned}
\operatorname{Pr}\left[\max _{i \in D_{m}} x_{i}<\Lambda-y\right] & =1-\exp \left(-\frac{\Lambda}{\sigma_{s d}^{2}}\right) \\
& +\sum_{n=1}^{2^{\| D m} \|}-1
\end{aligned}
$$

where $\left\|D_{m}\right\|$ is the number of the elements in decoding set $D_{m}, S_{m}(n)$ is the $n$-th non-empty sub-collection of the elements of $D_{m}$, and $\phi_{S_{m}(n)}$ is given by

$\phi_{S_{m}(n)}=\left\{\begin{array}{l}\frac{\Lambda}{\sigma_{s d}^{2}} \exp \left(-\frac{\Lambda}{\sigma_{s d}^{2}}\right), \sum_{i \in S_{m}(n)} \frac{1}{\sigma_{i d}^{2}}=\frac{1}{\sigma_{s d}^{2}} \\ \frac{\exp \left(-\sum_{i \in S_{m}(n)} \frac{\Lambda}{\sigma_{i d}^{2}}\right)-\exp \left(-\frac{\Lambda}{\sigma_{s d}^{2}}\right)}{1-\sum_{i \in S_{m}(n)} \frac{\sigma_{s d}^{2}}{\sigma_{i d}^{2}}}, \text { otherwise }\end{array}\right.$

Moreover, using Eq. (28) of [5], term $\operatorname{Pr}\left[\max _{i \in D_{m}} x_{i}<\Lambda-y+\right.$ $\left.z \gamma_{p} \Lambda\right]$ is derived as

$$
\operatorname{Pr}\left[\max _{i \in D_{m}} x_{i}<\Lambda-y+z \gamma_{p} \Lambda\right]=\mathrm{A}_{m}+\mathrm{B}_{m}
$$

wherein

$$
\mathrm{A}_{m}=\Xi+\sum_{n=1}^{2^{\left\|D_{m}\right\|}-1} \frac{(-1)^{\left\|S_{m}(n)\right\|} \Xi}{1+\sum_{i \in S_{m}(n)} \frac{\sigma_{p d}^{2} \gamma_{p} \Lambda}{\sigma_{i d}^{2}}} \exp \left(-\sum_{i \in S_{m}(n)} \frac{\Lambda}{\sigma_{i d}^{2}}\right)
$$

and

$\mathrm{B}_{m}=(1-\Xi)\left[1-\exp \left(-\frac{\Lambda}{\sigma_{s d}^{2}}\right)+\sum_{n=1}^{2^{\|D m\|}-1}(-1)^{\left\|S_{m}(n)\right\|} \phi_{S_{m}(n)}\right]$

where $\Xi=\frac{\sigma_{p d}^{2} \gamma_{p} \Lambda}{\sigma_{p d}^{2} \gamma_{p} \Lambda+\sigma_{s d}^{2}}$. In addition, from (1) and (29), the second term $\operatorname{Pr}\left[I_{\text {direct }}(k)<R, D=\emptyset \mid \hat{H}_{s}(k)=H_{0}\right]$ as given in the right-hand side of (32) is calculated as

$$
\begin{gathered}
\operatorname{Pr}\left[I_{\text {direct }}(k)<R, D=\emptyset \mid \hat{H}_{s}(k)=H_{0}\right] \\
=\pi_{1} \operatorname{Pr}\left[\left|h_{s d}(k)\right|^{2}<\Delta\right] \prod_{i=1}^{M} \operatorname{Pr}\left[\left|h_{s i}(k)\right|^{2}<\Lambda\right] \\
+\pi_{2} \operatorname{Pr}\left[\left|h_{s d}(k)\right|^{2}-\left|h_{p d}(k)\right|^{2} \gamma_{p} \Delta<\Delta\right] \\
\quad \times \prod_{i=1}^{M} \operatorname{Pr}\left[\left|h_{s i}(k)\right|^{2}-\left|h_{p i}(k)\right|^{2} \gamma_{p} \Lambda<\Lambda\right]
\end{gathered}
$$

where the probabilities $\operatorname{Pr}\left[\left|h_{s d}(k)\right|^{2}<\Delta\right]$ and $\operatorname{Pr}\left[\left|h_{s d}(k)\right|^{2}-\right.$ $\left.\left|h_{p d}(k)\right|^{2} \gamma_{p} \Delta<\Delta\right]$ are given by $1-\exp \left(-\frac{\Delta}{\sigma_{s d}^{2}}\right)$ and $1-$ $\frac{\sigma_{s d}^{2}}{\sigma_{p d}^{2} \gamma_{p} \Delta+\sigma_{s d}^{2}} \exp \left(-\frac{\Delta}{\sigma_{s d}^{2}}\right)$, respectively.

2) ACK Based Selective Best-Relay Cooperation: This scheme typically adopts the best-relay transmission mode only when the decoding set is not empty and CD acknowledges its failure of decoding CS' signal through the direct link. Thus, an outage probability of the ACK based selective best-relay cooperation scheme is given by

Pout $_{\mathrm{ACK}}$, multiple

$$
\begin{aligned}
= & \sum_{m=1}^{2^{M}-1} \operatorname{Pr}\left[I_{\text {best-relay }}(k)<R, D=D_{m}, I_{\text {direct }}(k)<R \mid \hat{H}_{s}(k)=H_{0}\right] \\
& +\operatorname{Pr}\left[I_{\text {direct }}(k)<R, D=\emptyset \mid \hat{H}_{s}(k)=H_{0}\right]
\end{aligned}
$$

Using (1), (29) and (31), we can expand the term $\operatorname{Pr}\left[I_{\text {best-relay }}(k)<R, D=D_{m}, I_{\text {direct }}(k)<R \mid \hat{H}_{s}(k)=H_{0}\right]$ as Eq. (38) at the top of the following page, where the probabilities $\operatorname{Pr}\left[\left|h_{s i}(k)\right|^{2}>\Lambda\right]$ and $\operatorname{Pr}\left[\left|h_{s i}(k)\right|^{2}-\left|h_{p i}(k)\right|^{2} \gamma_{p} \Lambda>\Lambda\right]$ are easily calculated as $\exp \left(-\frac{\Lambda}{\sigma_{s i}^{2}}\right)$ and $\frac{\sigma_{s i}^{2}}{\sigma_{p i}^{2} \gamma_{p} \Lambda+\sigma_{s i}^{2}} \exp \left(-\frac{\Lambda}{\sigma_{s i}^{2}}\right)$, respectively. Moreover, terms $\operatorname{Pr}\left[\max _{i \in D_{m}}\left|h_{i d}(k)\right|^{2}<\Lambda-\right.$ $\left.\left|h_{s d}(k)\right|^{2},\left|h_{s d}(k)\right|^{2}<\Delta\right]$ and $\operatorname{Pr}\left[\max _{i \in D_{m}}\left|h_{i d}(k)\right|^{2}<\Lambda-\right.$ $\left.\left|h_{s d}(k)\right|^{2}+\left|h_{p d}(k)\right|^{2} \gamma_{p} \Lambda,\left|h_{s d}(k)\right|^{2}<\Delta+\left|h_{p d}(k)\right|^{2} \gamma_{p} \Delta\right]$ are determined in closed-form by (B.2) and (B.3), respectively, in Appendix B. In addition, a closed-form solution to the term $\operatorname{Pr}\left[I_{\text {direct }}(k)<R, D=\emptyset \mid \hat{H}_{s}(k)=H_{0}\right]$ in the right-hand side of (37) is given by (36).

3) Numerical Results: Fig. 8 shows the outage probability versus the primary and secondary SNRs $\left(\gamma_{p}\right.$ and $\left.\gamma_{s}\right)$ of the non-cooperation and the non-ACK and ACK based selective best-relay cooperation schemes by using (9), (32) and (37). As shown in Fig. 8, both the non-ACK and ACK based best-relay cooperation schemes outperform the traditional noncooperative direct transmission. Moreover, the outage probability floors of the non-ACK and ACK based selective cooperation schemes are lower than that of the non-cooperation. As the number of cognitive relays increases, the outage floors 


$$
\begin{aligned}
& \operatorname{Pr}\left[I_{\text {best-relay }}(k)<R, D=D_{m}, I_{\text {direct }}(k)<R \mid \hat{H}_{s}(k)=H_{0}\right] \\
& =\pi_{1} \operatorname{Pr}\left[\max _{i \in D_{m}}\left|h_{i d}(k)\right|^{2}<\Lambda-\left|h_{s d}(k)\right|^{2},\left|h_{s d}(k)\right|^{2}<\Delta\right] \prod_{i \in D_{m}} \operatorname{Pr}\left[\left|h_{s i}(k)\right|^{2}>\Lambda\right] \prod_{j \in \bar{D}_{m}} \operatorname{Pr}\left[\left|h_{s j}(k)\right|^{2}<\Lambda\right] \\
& \left.+\pi_{2} \operatorname{Pr} \max _{i \in D_{m}}\left|h_{i d}(k)\right|^{2}<\Lambda-\left|h_{s d}(k)\right|^{2}+\left|h_{p d}(k)\right|^{2} \gamma_{p} \Lambda,\left|h_{s d}(k)\right|^{2}<\Delta+\left|h_{p d}(k)\right|^{2} \gamma_{p} \Delta\right] \\
& \quad \times \prod_{i \in D_{m}} \operatorname{Pr}\left[\left|h_{s i}(k)\right|^{2}-\left|h_{p i}(k)\right|^{2} \gamma_{p} \Lambda>\Lambda\right] \prod_{j \in \bar{D}_{m}} \operatorname{Pr}\left[\left|h_{s j}(k)\right|^{2}-\left|h_{p j}(k)\right|^{2} \gamma_{p} \Lambda<\Lambda\right]
\end{aligned}
$$

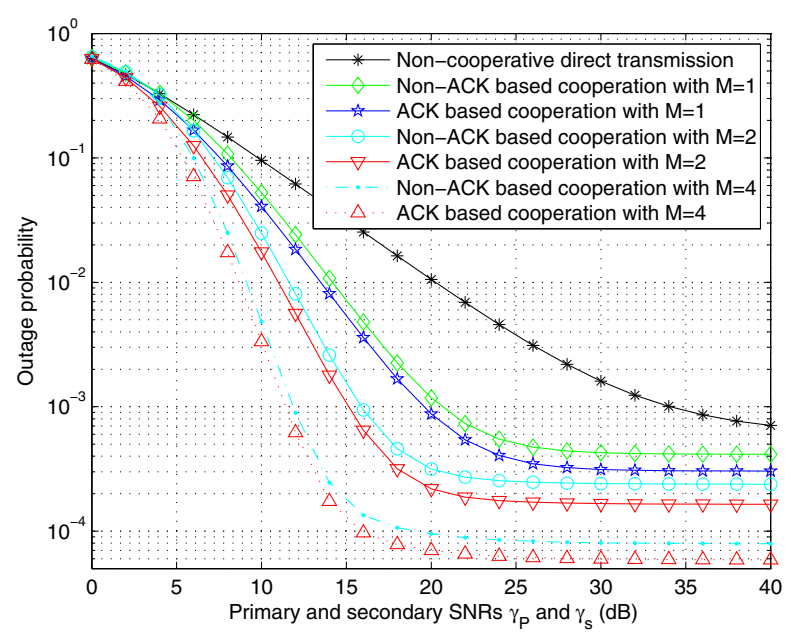

Fig. 8. Outage probability versus primary and secondary SNRs $\gamma_{p}$ and $\gamma_{s}$ of the non-cooperative transmission and selective best-relay cooperation schemes for different number of cognitive relays with $P_{a}=0.6, P_{d}=0.99$, $P_{f}=0.01, R=1 \mathrm{bit} / \mathrm{s} / \mathrm{Hz}, \sigma_{p i}^{2}=\sigma_{p d}^{2}=0.1$, and $\sigma_{s i}^{2}=\sigma_{i d}^{2}=\sigma_{s d}^{2}=1$.

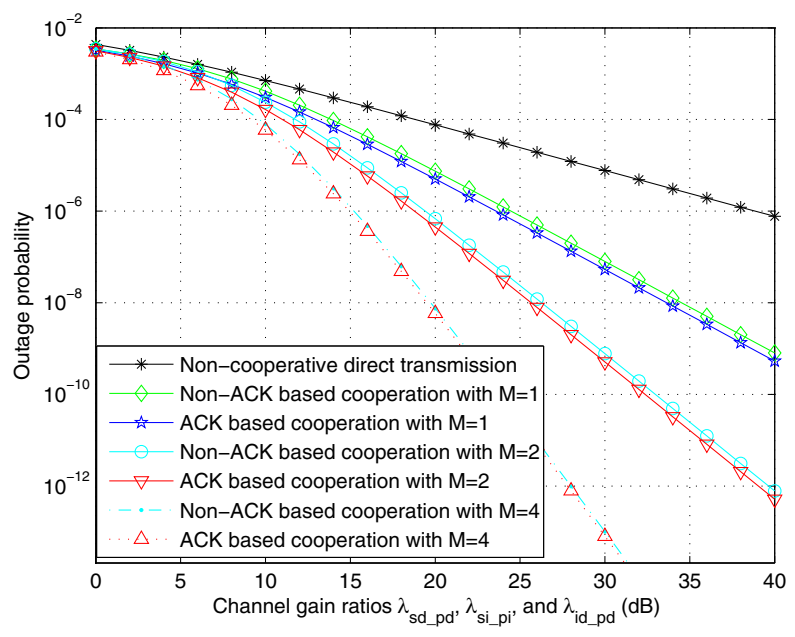

Fig. 9. Outage probability versus the channel gain ratios $\left(\lambda_{s d-p d}=\right.$ $\sigma_{s d}^{2} / \sigma_{p d}^{2}, \lambda_{s i-p i}=\sigma_{s i}^{2} / \sigma_{p i}^{2}$, and $\left.\lambda_{i d-p d}=\sigma_{i d}^{2} / \sigma_{p d}^{2}\right)$ of the non-cooperation and the best-relay cooperation schemes with $P_{a}=0.6, P_{d}=0.99$, $P_{f}=0.01, \gamma_{s}=\gamma_{p}=30 \mathrm{~dB}$, and $R=1 \mathrm{bit} / \mathrm{s} / \mathrm{Hz}$.

of the non-ACK and ACK based cooperation schemes are reduced greatly, which further shows the advantage of the selective best-relay cooperation. In addition, one can see from Fig. 8 that the ACK based selective best-relay cooperation scheme performs better than the non-ACK based scheme in terms of the outage probability.

Fig. 9 plots (9), (32) and (37) as a function of the channel gain ratios, $\lambda_{s d-p d}=\sigma_{s d}^{2} / \sigma_{p d}^{2}, \lambda_{s i-p i}=\sigma_{s i}^{2} / \sigma_{p i}^{2}$, and $\lambda_{i d-p d}=\sigma_{i d}^{2} / \sigma_{p d}^{2}$, with relatively high primary and secondary
SNRs (i.e., $\gamma_{s}=\gamma_{p}=30 \mathrm{~dB}$ ). As shown in Fig. 9, both the non-ACK and ACK based selective best-relay cooperation schemes outperform the traditional non-cooperation scheme in terms of the outage probability. It is worth mentioning that, in high channel gain ratio regions, the slopes of the outage probability curves corresponding to the non-ACK and ACK based selective best-relay cooperation are much larger than that of the non-cooperation scheme, which further increase with an increasing number of cognitive relays. This implies that, as the channel gain ratios increase, the outage probabilities of the non-ACK and ACK based cooperation scheme are improved much faster than that of the traditional noncooperation scheme.

\section{Diversity-Multiplexing Tradeoff Analysis}

In this section, we examine the DMT for the proposed non-ACK and ACK based selective best-relay cooperation schemes. From Lemma 3 as given in Appendix C, a DMT of the non-ACK based selective best-relay cooperation scheme is obtained as

$$
d_{\text {non-ACK,multiple }}+2(M+1) r=M+1
$$

which shows that a maximum diversity gain $M+1$ is achieved as $r \rightarrow 0$ and a maximum multiplexing gain $1 / 2$ is achieved as $d_{\text {non-ACK,mutiple }} \rightarrow 0$. In addition, using the results of Lemma 4 in Appendix C, we obtain a DMT of the ACK based selective best-relay cooperation scheme as

$$
d_{\mathrm{ACK}, \text { multiple }}+(2 M+1) r=M+1
$$

One can observe from (40) that a maximum multiplexing gain $(M+1) /(2 M+1)$ is achieved as the diversity gain approaches zero, showing the advantage of the ACK based selective bestrelay cooperation scheme over the non-ACK based scheme. Moreover, with an increasing number of cognitive relays $M$, the maximum multiplexing gain of the ACK based selective best-relay cooperation decreases and eventually becomes onehalf when $M \rightarrow \infty$.

Fig. 10 compares the DMTs of the non-cooperation and the non-ACK and ACK based best-relay cooperation schemes. One can observe from Fig. 10 that the maximum diversity gains of the non-ACK and ACK based selective best-relay cooperation scheme with $M=2$ and 4 are $d=3$ and 5 , respectively, which are larger than that of the non-cooperative transmission. Fig. 10 also shows that the ACK based selective best-relay cooperation outperforms the non-ACK based cooperation in terms of the DMT performance. In addition, one can see from Fig. 10 that, as the number of cognitive relays increases from $M=2$ to 4 , the maximum multiplexing gains achieved by the ACK based cooperation schemes decreases from $r=3 / 5$ to $5 / 9$. 


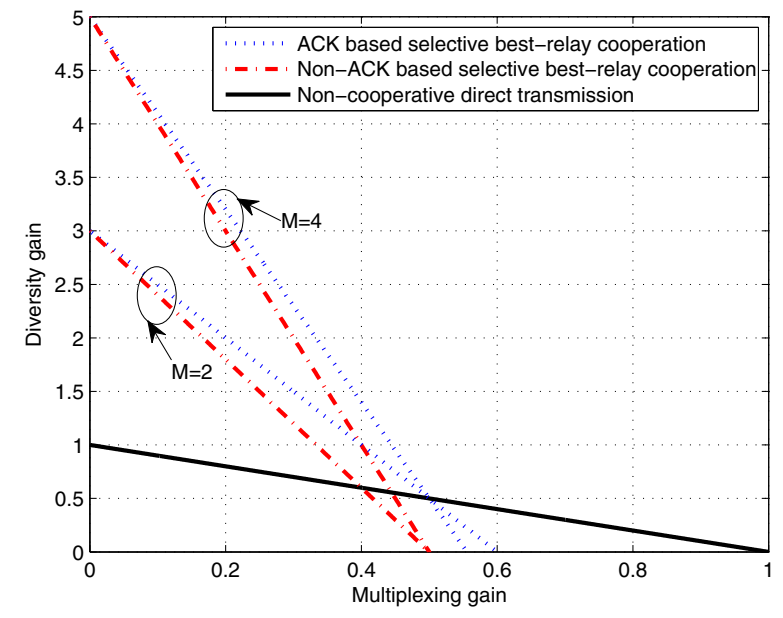

Fig. 10. DMTs of the non-cooperative direct transmission and the nonACK and ACK based selective best-relay cooperation schemes with different number of cognitive relays.

\section{CONCLUSION}

In this paper, we investigated a selective cooperation framework for secondary data transmissions in cognitive radio networks. We studied non-ACK and ACK based selective cooperation schemes and derived closed-form outage probability expressions for the two selective cooperation schemes in Rayleigh fading channels. We showed that an outage probability floor of the secondary transmissions occurs in high SNR regions and, moreover, the outage floors of the non$\mathrm{ACK}$ and ACK based selective cooperation are lower than that of the traditional direct transmissions. We proposed a generalized DMT definition and developed DMTs of the nonACK and ACK based selective cooperation schemes. We then extended the selective cooperation to multiple-relay cognitive radio networks and explored a selective best-relay cooperation by considering the best cognitive relay only to assist the secondary transmissions. We examined non-ACK and ACK based selective best-relay cooperation schemes and evaluated their performance in terms of outage probability and DMT.

\section{APPENDIX A}

\section{Asymptotic Outage Analysis of the Selective COOPERATION}

In this Appendix, we examine an asymptotic outage probability analysis in high SNR regions for the proposed nonACK and ACK based selective cooperation schemes. At the beginning, let us develop Theorem 1 that would be frequently used for the asymptotic outage analysis throughout this paper. Theorem 1: Given any positive a, two independent exponential random variables $x$ and $y$ (with means $\sigma_{x}^{2}$ and $\sigma_{y}^{2}$, respectively) and $x-a y<0$, the following equation holds for $\lambda=\sigma_{x}^{2} / \sigma_{y}^{2} \rightarrow \infty$

$$
1-\exp \left(\frac{x-a y}{b \sigma_{x}^{2}}\right)=-\frac{x-a y}{b \sigma_{x}^{2}}+O\left(\frac{x-a y}{b \sigma_{x}^{2}}\right)
$$

where $b$ is any positive value and $O(\cdot)$ represents a higherorder infinitesimal.

Proof: Without loss of generality, by letting $t=x-a y$, a conditional probability density function (PDF) of $t$ given $t<0$ is obtained as

$$
f(t \mid t<0)=\frac{1}{a \sigma_{y}^{2}} \exp \left(\frac{t}{a \sigma_{y}^{2}}\right)
$$

From (A.1), the mean and variance of random variable $t$ given $t<0$ are found as $-a \sigma_{y}^{2}$ and $a^{2} \sigma_{y}^{4}$, respectively. Hence, given $t<0$, we can easily obtain the mean and variance of $\frac{x-a y}{b \sigma_{x}^{2}}$ as $-\frac{a}{b \lambda}$ and $\frac{a^{2}}{b^{2} \lambda^{2}}$, respectively, which shows that both the mean and variance approach zero as $\lambda \rightarrow \infty$. This implies that random variable $\frac{x-a y}{b \sigma_{x}^{2}}$ approaches zero with probability 1 for $\lambda \rightarrow \infty$. Accordingly, applying Taylor series expansion to $\exp \left(\frac{x-a y}{b \sigma_{x}^{2}}\right)$ yields

$$
1-\exp \left(\frac{x-a y}{b \sigma_{x}^{2}}\right)=-\frac{x-a y}{b \sigma_{x}^{2}}+O\left(\frac{x-a y}{b \sigma_{x}^{2}}\right)
$$

The proof is completed.

\section{A. Lemma 1 (Non-ACK Based Selective Cooperation Scheme)}

This lemma presents an asymptotic outage analysis for the non-ACK based selective cooperation in high SNR regions. Considering $\gamma_{p}=\alpha \gamma_{s}$ and letting $\gamma_{s} \rightarrow+\infty$, we can easily obtain $\lim _{\gamma_{s} \rightarrow \infty} \operatorname{Pr}\left[\left|h_{s d}(k)\right|^{2}+\right.$ $\left.\left|h_{r d}(k)\right|^{2}<\Lambda\right]=0, \lim _{\gamma_{s} \rightarrow \infty} \operatorname{Pr}\left[\left|h_{s r}(k)\right|^{2}-\left|h_{p r}(k)\right|^{2} \gamma_{p} \Lambda>\right.$ $\Lambda]=\frac{1}{\lambda_{s r-p r}^{-1} v+1}, \lim _{\gamma_{s} \rightarrow \infty} \operatorname{Pr}\left[\left|h_{s d}(k)\right|^{2}<\Delta\right]=0$, $\lim _{\gamma_{s} \rightarrow \infty} \operatorname{Pr}\left[\left|h_{s d}(k)\right|^{2}-\left|h_{p d}(k)\right|^{2} \gamma_{p} \Delta<\Delta\right]=\frac{\alpha}{\lambda_{s d-p d}^{-1} \nu+1} \cdot \frac{2^{R}-1}{\lambda_{s d-p d}}$, and $\lim _{\gamma_{s} \rightarrow \infty} \operatorname{Pr}\left[\left|h_{s r}(k)\right|^{2}-\left|h_{p r}(k)\right|^{2} \gamma_{p} \Lambda<\Lambda\right]=\frac{\alpha}{\lambda_{s r-p r}^{-1} v+1}$. $\frac{2^{2 R}-1}{\lambda_{s r-p r}}$, where $v=\alpha\left(2^{2 R}-1\right), \nu=\alpha\left(2^{R}-1\right)$, and $\lambda_{s r-p r}=\sigma_{s r}^{2} / \sigma_{p r}^{2}$. Using these results and (13), an asymptotic outage probability (called outage probability floor) of the nonACK based selective cooperation is given by

$$
\begin{aligned}
& \text { Pout } t_{\text {non-ACK,floor }} \\
& =\lim _{\gamma_{s} \rightarrow \infty} \text { Pout }_{\text {non-ACK }} \\
& =\frac{\pi_{2}}{\lambda_{s r-p r}^{-1} v+1} \\
& \quad \times \lim _{\gamma_{s} \rightarrow \infty} \operatorname{Pr}\left[\left|h_{s d}(k)\right|^{2}+\left|h_{r d}(k)\right|^{2}-\left|h_{p d}(k)\right|^{2} \gamma_{p} \Lambda<0\right] \\
& +\frac{\pi_{2} \alpha^{2}}{\left(\lambda_{s d-p d}^{-1} \nu+1\right)\left(\lambda_{s r-p r}^{-1} v+1\right)} \cdot \frac{\left(2^{R}-1\right)\left(2^{2 R}-1\right)}{\lambda_{s d-p d} \lambda_{s r-p r}}
\end{aligned}
$$

By denoting $x=\left|h_{r d}(k)\right|^{2}, y=\left|h_{s d}(k)\right|^{2}$ and $z=\left|h_{p d}(k)\right|^{2}$, term $\lim _{\gamma_{s} \rightarrow \infty} \operatorname{Pr}\left[\left|h_{s d}(k)\right|^{2}+\left|h_{r d}(k)\right|^{2}-\left|h_{p d}(k)\right|^{2} \gamma_{p} \Lambda<0\right]$ inside (A.3) can be rewritten as $\lim _{\gamma_{s} \rightarrow \infty} \operatorname{Pr}\left[x<-y+z \gamma_{p} \Lambda\right]$, which is calculated as

$$
\begin{aligned}
& \lim _{\gamma_{s} \rightarrow \infty} \operatorname{Pr}\left[x<-y+z \gamma_{p} \Lambda\right] \\
& =\iint_{0<-y+z \gamma_{p} \Lambda}\left[1-\exp \left(-\frac{-y+z \gamma_{p} \Lambda}{\sigma_{r d}^{2}}\right)\right] \\
& \quad \times \frac{1}{\sigma_{s d}^{2} \sigma_{p d}^{2}} \exp \left(-\frac{y}{\sigma_{s d}^{2}}-\frac{z}{\sigma_{p d}^{2}}\right) d y d z
\end{aligned}
$$

Considering $\lambda_{r d-p d} \rightarrow \infty$ and following Theorem 1 , term $1-$ $\exp \left(-\frac{-y+z \gamma_{p} \Lambda}{\sigma_{r d}^{2}}\right)$ can be approximated to $\left(-y+z \gamma_{p} \Lambda\right) / \sigma_{r d}^{2}$. 
Substituting this result into (A.4) and performing the double integral yields,

$\lim _{\gamma_{s} \rightarrow \infty} \operatorname{Pr}\left[x<-y+z \gamma_{p} \Lambda\right]=\frac{\alpha^{2}}{\left(\lambda_{s d-p d}^{-1} v+1\right)} \cdot \frac{\left(2^{2 R}-1\right)^{2}}{\lambda_{s d-p d} \lambda_{r d-p d}}$

where $\lambda_{s d-p d}=\sigma_{s d}^{2} / \sigma_{p d}^{2}$ and $\lambda_{r d-p d}=\sigma_{r d}^{2} / \sigma_{p d}^{2}$. Denoting $\rho=\lambda_{s r-p r} / \lambda_{s d-p d}$ and $\delta=\lambda_{r d-p d} / \lambda_{s d-p d}$ and substituting (A.5) into (A.3), we obtain

$$
\begin{aligned}
\text { Pout }_{\text {non-ACK, floor }}= & \frac{\pi_{2} \alpha^{2}}{\left(\rho^{-1} \lambda_{s d-p d}^{-1} v+1\right)\left(\lambda_{s d-p d}^{-1} v+1\right) \delta} \\
& \times\left(\frac{2^{2 R}-1}{\lambda_{s d-p d}}\right)^{2} \\
& +\frac{\pi_{2} \alpha^{2}}{\left(\lambda_{s d-p d}^{-1} \nu+1\right)\left(\lambda_{s d-p d}^{-1} v+\rho\right)} \\
& \times \frac{\left(2^{2 R}-1\right)\left(2^{R}-1\right)}{\lambda_{s d-p d}^{2}}
\end{aligned}
$$

\section{B. Lemma 2 (ACK Based Selective Cooperation Scheme)}

Letting $\gamma_{p}=\alpha \gamma_{s}$ and considering $\gamma_{s} \rightarrow \infty$, we can easily obtain that both $\operatorname{Pr}\left[\left|h_{s d}(k)\right|^{2}+\left|h_{r d}(k)\right|^{2}<\Lambda,\left|h_{s d}(k)\right|^{2}<\right.$ $\Delta]$ and $\operatorname{Pr}\left[\left|h_{s d}(k)\right|^{2}<\Delta\right]$ are equal to zero. Also, it is easy to show $\lim _{\gamma_{s} \rightarrow \infty} \operatorname{Pr}\left[\left|h_{s r}(k)\right|^{2}-\left|h_{p r}(k)\right|^{2} \gamma_{p} \Lambda>\Lambda\right]=$ $1 /\left(\lambda_{s r-p r}^{-1} v+1\right), \lim _{\gamma_{s} \rightarrow \infty} \operatorname{Pr}\left[\left|h_{s d}(k)\right|^{2}-\left|h_{p d}(k)\right|^{2} \gamma_{p} \Delta<\Delta\right]=$ $\alpha\left(2^{R}-1\right) /\left[\lambda_{s d-p d}\left(\lambda_{s d-p d}^{-1} \nu+1\right)\right]$, and $\lim _{\gamma_{s} \rightarrow \infty} \operatorname{Pr}\left[\left|h_{s r}(k)\right|^{2}-\right.$ $\left.\left|h_{p r}(k)\right|^{2} \gamma_{p} \Lambda<\Lambda\right]=\alpha\left(2^{2 R}-1\right) /\left[\lambda_{s r-p r}\left(\lambda_{s r-p r}^{-1} v+1\right)\right]$, wherein $\lambda_{s d-p d}=\sigma_{s d}^{2} / \sigma_{p d}^{2}, \lambda_{s r-p r}=\sigma_{s r}^{2} / \sigma_{p r}^{2}$ and $v=$ $\alpha\left(2^{2 R}-1\right)$. By substituting these results into (18) and denoting $x=\left|h_{r d}(k)\right|^{2}, y=\left|h_{s d}(k)\right|^{2}$ and $z=\left|h_{p d}(k)\right|^{2}$, an outage probability floor of the proposed ACK based selective cooperation scheme can be expressed as

$$
\begin{aligned}
\text { Pout }_{\mathrm{ACK}, \text { floor }}= & \frac{\pi_{2}}{\lambda_{s r-p r}^{-1} v+1} \\
& \times \lim _{\gamma_{s} \rightarrow \infty} \operatorname{Pr}[x<-y+z v, 0<-y+z \nu] \\
& +\frac{\pi_{2} \alpha^{2}}{\left(\lambda_{s d-p d}^{-1} \nu+1\right)\left(\lambda_{s r-p r}^{-1} v+1\right)} \\
& \times \frac{\left(2^{R}-1\right)\left(2^{2 R}-1\right)}{\lambda_{s d-p d} \lambda_{s r-p r}}
\end{aligned}
$$

where term $\lim _{\gamma_{s} \rightarrow \infty} \operatorname{Pr}[x<-y+z v, 0<-y+z \nu]$ is given by

$$
\begin{aligned}
& \lim _{\gamma_{s} \rightarrow \infty} \operatorname{Pr}[x<-y+z v, 0<-y+z \nu] \\
&=\iint_{0<-y+z \nu}\left[1-\exp \left(-\frac{-y+z v}{\sigma_{r d}^{2}}\right)\right] \\
& \quad \times \frac{1}{\sigma_{s d}^{2} \sigma_{p d}^{2}} \exp \left(-\frac{y}{\sigma_{s d}^{2}}-\frac{z}{\sigma_{p d}^{2}}\right) d y d z
\end{aligned}
$$

since $-y+z v>0$ if $-y+z \nu>0$ (due to $v>\nu$ ). Considering $\lambda_{r d-p d} \rightarrow \infty$ and following Theorem 1 , term $1-\exp \left(-\frac{-y+z v}{\sigma_{r d}^{2}}\right)$ inside the integral of the preceding equation can be approximated to $(-y+z v) / \sigma_{r d}^{2}$. Thus, (A.8) can be given by

$$
\begin{aligned}
& \lim _{\gamma_{s} \rightarrow \infty} \operatorname{Pr}[x<-y+z v, 0<-y+z \nu] \\
& =\frac{\sigma_{s d}^{2} \sigma_{p d}^{4} v \nu}{\sigma_{r d}^{2}\left(\sigma_{p d}^{2} \nu+\sigma_{s d}^{2}\right)^{2}}+\frac{\sigma_{p d}^{4} v \nu}{\sigma_{r d}^{2}\left(\sigma_{p d}^{2} \nu+\sigma_{s d}^{2}\right)} \\
& \quad+\frac{\sigma_{s d}^{2} \sigma_{p d}^{4} \nu^{2}}{\sigma_{r d}^{2}\left(\sigma_{p d}^{2} \nu+\sigma_{s d}^{2}\right)^{2}}
\end{aligned}
$$

Substituting (A.9) into (A.7) yields

$$
\begin{aligned}
\text { Pout }_{\mathrm{ACK}, \text { floor }}= & \frac{\pi_{2} \alpha^{2}}{\left(\lambda_{s r-p r}^{-1} v+1\right)\left(\lambda_{s d-p d}^{-1} \nu+1\right)^{2}} \\
& \times \frac{\left(2^{R}-1\right)\left(2^{2 R}-2^{R}\right)}{\lambda_{s d-p d} \lambda_{r d-p d}} \\
+ & \frac{\pi_{2} \alpha^{2}}{\left(\lambda_{s r-p r}^{-1} v+1\right)\left(\lambda_{s d-p d}^{-1} \nu+1\right)} \\
& \times \frac{\left(2^{R}-1\right)\left(2^{2 R}-1\right)}{\lambda_{s d-p d} \lambda_{r d-p d}} \\
+ & \frac{\pi_{2} \alpha^{2}}{\left(\lambda_{s d-p d}^{-1} \nu+1\right)\left(\lambda_{s r-p r}^{-1} v+1\right)} \\
& \times \frac{\left(2^{R}-1\right)\left(2^{2 R}-1\right)}{\lambda_{s d-p d} \lambda_{s r-p r}}
\end{aligned}
$$

which completes Lemma 2.

\section{APPENDIX B}

\section{Probability Derivation of THE ACK BASED SElEctive Best-Relay COOPERATION}

Denoting $x_{i}=\left|h_{i d}(k)\right|^{2}$ and $y=\left|h_{s d}(k)\right|^{2}$, one can rewrite $\operatorname{Pr}\left[\max _{i \in D_{m}}\left|h_{i d}(k)\right|^{2}<\Lambda-\left|h_{s d}(k)\right|^{2},\left|h_{s d}(k)\right|^{2}<\Delta\right]$ as $\operatorname{Pr}\left[\max _{i \in D_{m}} x_{i}<\Lambda-y, 0<\Delta-y\right]$, which is calculated as

$$
\begin{aligned}
& \operatorname{Pr}\left[\max _{i \in D_{m}} x_{i}<\Lambda-y, 0<\Delta-y\right] \\
& =\int_{0}^{\Delta} \frac{1}{\sigma_{s d}^{2}} \exp \left(-\frac{y}{\sigma_{s d}^{2}}\right) \prod_{i \in D_{m}}\left[1-\exp \left(-\frac{\Lambda-y}{\sigma_{i d}^{2}}\right)\right] d y
\end{aligned}
$$

By using the binomial expansion formula, term $\prod_{i \in D_{m}}\left[1-\exp \left(-\frac{\Lambda-y}{\sigma_{i d}^{2}}\right)\right]$ can be expanded as $1+\sum_{n=1}^{2^{\left\|D_{m}\right\|}-1}(-1)^{\left\|S_{m}(n)\right\|} \exp \left(-\sum_{i \in S_{m}(n)} \frac{\Lambda-y}{\sigma_{i d}^{2}}\right), \quad$ where $\left\|D_{m}\right\|$ represents the number of the elements in decoding set $D_{m}$ and $S_{m}(n)$ is the $n$-th non-empty subcollection of the elements in $D_{m}$. Substituting this result into the preceding equation and performing integration yield

$$
\begin{aligned}
& \operatorname{Pr}\left[\max _{i \in D_{m}} x_{i}<\Lambda-y, 0<\Delta-y\right] \\
& =1-\exp \left(-\frac{\Delta}{\sigma_{s d}^{2}}\right)+\sum_{n=1}^{2^{\left\|D_{m}\right\|}-1}(-1)^{\left\|S_{m}(n)\right\|} \varphi_{S_{m}(n)}
\end{aligned}
$$


where $\varphi_{S_{m}(n)}$ is given by

$$
\varphi_{S_{m}(n)}=\left\{\begin{array}{l}
\frac{\Delta}{\sigma_{s d}^{2}} \exp \left(-\frac{\Lambda}{\sigma_{s d}^{2}}\right), \frac{1}{\sigma_{s d}^{2}}=\sum_{i \in S_{m}(n)} \frac{1}{\sigma_{i d}^{2}} \\
\frac{\exp \left(-\sum_{i \in S_{m}(n)} \frac{\Lambda}{\sigma_{i d}^{2}}\right)}{1-\sum_{i \in S_{m}(n)} \frac{\sigma_{s d}^{2}}{\sigma_{i d}^{2}}} \\
\times\left[1-\exp \left(-\frac{\Delta}{\sigma_{s d}^{2}}+\sum_{i \in S_{m}(n)} \frac{\Delta}{\sigma_{i d}^{2}}\right)\right]
\end{array},\right. \text { otherwise }
$$

Similarly, by letting $x_{i}=\left|h_{i d}(k)\right|^{2}, y=\left|h_{s d}(k)\right|^{2}$ and $z=\left|h_{p d}(k)\right|^{2}$, term $\operatorname{Pr}\left[\max _{i \in D_{m}}\left|h_{i d}(k)\right|^{2}<\Lambda-\left|h_{s d}(k)\right|^{2}+\right.$ $\left.\left|h_{p d}(k)\right|^{2} \gamma_{p} \Lambda,\left|h_{s d}(k)\right|^{2}<\Delta+\left|h_{p d}(k)\right|^{2} \gamma_{p} \Delta\right]$ can be equivalently expressed as $\operatorname{Pr}\left[\max _{i \in D_{m}} x_{i}<\Lambda-y+z \gamma_{p} \Lambda, 0<\right.$ $\left.\Delta-y+z \gamma_{p} \Delta\right]$. Since random variables $x_{i}, y$ and $z$ follow exponential distributions and are independent of eath other, we can obtain

$$
\begin{aligned}
& \operatorname{Pr}\left[\max _{i \in D_{m}} x_{i}<\Lambda-y+z \gamma_{p} \Lambda, 0<\Delta-y+z \gamma_{p} \Delta\right] \\
& =1-\frac{\sigma_{s d}^{2}}{\sigma_{s d}^{2}+\sigma_{p d}^{2} \gamma_{p} \Delta} \exp \left(-\frac{\Delta}{\sigma_{s d}^{2}}\right)+\sum_{n=1}^{2^{\left\|D_{m}\right\|}-1}(-1)^{\left\|S_{m}(n)\right\|} \psi_{S_{m}(n)}
\end{aligned}
$$

where $\psi_{S_{m}(n)}$ is given by

$$
\psi_{S_{m}(n)}=\frac{\Delta}{\sigma_{s d}^{2}+\sigma_{p d}^{2} \gamma_{p} \Lambda}\left(1+\frac{\sigma_{s d}^{2} \sigma_{p d}^{2} \gamma_{p}}{\sigma_{s d}^{2}+\sigma_{p d}^{2} \gamma_{p} \Lambda}\right) \exp \left(-\frac{\Lambda}{\sigma_{s d}^{2}}\right)
$$

for $\frac{1}{\sigma_{s d}^{2}}=\sum_{i \in S_{m}(n)} \frac{1}{\sigma_{i d}^{2}}$. For the case of $\frac{1}{\sigma_{s d}^{2}} \neq \sum_{i \in S_{m}(n)} \frac{1}{\sigma_{i d}^{2}}$, $\psi_{S_{m}(n)}$ is obtained as Eq. (B.5) at the top of the following page.

\section{APPENDIX C}

\section{DMT Analysis of the SElEctive Best-Relay COOPERATION}

This Appendix develops DMTs of both the non-ACK and ACK based selective best-relay cooperation schemes.

\section{A. Lemma 3 (Non-ACK Based Selective Best-Relay Coopera- tion Scheme)}

Similar to (22), the diversity gain of the non-ACK based selective best-relay cooperation scheme is defined as

$$
d_{\text {non-ACK,multiple }}=-\lim _{\lambda_{s d-p d} \rightarrow \infty} \frac{\log \left(\text { Pout }_{\text {non-ACK,multiple }}\right)}{\log \left(\lambda_{s d-p d}\right)}
$$

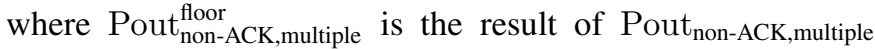
as the primary and secondary SNRs $\left(\gamma_{p}\right.$ and $\left.\gamma_{s}\right)$ approach infinity. Denoting $\gamma_{p}=\alpha \gamma_{s}$ and considering $\gamma_{s} \rightarrow \infty$, an outage probability floor Pout $t_{\text {non-ACK,multiple }}$ is calculated from (32) as

$$
\begin{aligned}
& \text { Pout } \text { floor-ACK, multiple }_{\text {flon-A }}^{2^{M}-1}{ }_{=} \sum_{m=1} \lim _{\gamma_{s} \rightarrow \infty} \operatorname{Pr}\left[I_{\text {best-relay }}(k)<R, D=D_{m} \mid \hat{H}_{s}(k)=H_{0}\right] \\
& \quad+\lim _{\gamma_{s} \rightarrow \infty} \operatorname{Pr}\left[I_{\text {direct }}(k)<R, D=\emptyset \mid \hat{H}_{s}(k)=H_{0}\right]
\end{aligned}
$$

From (35), we have

$$
\begin{aligned}
& \lim _{\gamma_{s} \rightarrow \infty} \operatorname{Pr}\left[\max _{i \in D_{m}}\left|h_{i d}(k)\right|^{2}<\Lambda-\left|h_{s d}(k)\right|^{2}+\left|h_{p d}(k)\right|^{2} \gamma_{p} \Lambda\right] \\
& =\lim _{\gamma_{s} \rightarrow \infty} \mathrm{A}_{m}+\lim _{\gamma_{s} \rightarrow \infty} \mathrm{B}_{m}
\end{aligned}
$$

where $\quad \mathrm{A}_{m}=\int_{-\infty}^{0} \frac{\Psi}{\sigma_{p d}^{2} \gamma_{p} \Lambda+\sigma_{s d}^{2}} \exp \left(\frac{t}{\sigma_{p d}^{2} \gamma_{p} \Lambda}\right) d t$ and $\quad \mathrm{B}_{m}=\int_{0}^{\Lambda} \frac{\Psi}{\sigma_{s d}^{2}+\sigma_{p d}^{2} \gamma_{p} \Lambda} \exp \left(-\frac{t}{\sigma_{s d}^{2}}\right) d t$, wherein $\Psi=\prod_{i \in D_{m}}\left[1-\exp \left(-\frac{\Lambda-t}{\sigma_{i d}^{2}}\right)\right]$. Denoting $\lambda_{s d-p d}=\sigma_{s d}^{2} / \sigma_{p d}^{2}$, $\lambda_{i d-p d}=\sigma_{i d}^{2} / \sigma_{p d}^{2}$ and $\delta_{i}=\lambda_{i d-p d} / \lambda_{s d-p d}$, and considering $\left(\lambda_{s d-p d}, \gamma_{s}\right) \rightarrow \infty$, term $\Psi$ can be approximated to $\Psi=\frac{(-1)^{\left\|D_{m}\right\|}}{\prod_{i \in D_{m}} \sigma_{i d}^{2}} \cdot t^{\left\|D_{m}\right\|}$. Substituting this result into $\mathrm{A}_{m}$ yields

$$
\lim _{\gamma_{s} \rightarrow \infty} \mathrm{A}_{m}=\frac{\alpha^{\left\|D_{m}\right\|+1} v\left\|D_{m}\right\| !}{\left(\lambda_{s d-p d}^{-1} v+1\right) \prod_{i \in D_{m}} \delta_{i}} \cdot\left(\frac{2^{2 R}-1}{\lambda_{s d-p d}}\right)^{\left\|D_{m}\right\|+1}
$$

wherein $\delta_{i}=\lambda_{i d-p d} / \lambda_{s d-p d}$ and $\lambda_{i d-p d}=\sigma_{i d}^{2} / \sigma_{p d}^{2}$. We can also obtain $\lim _{\gamma_{s} \rightarrow \infty} \mathrm{B}_{m}=0$. Meanwhile, one can easily have $\lim _{\gamma_{s} \rightarrow \infty} \operatorname{Pr}\left[\max _{i \in D_{m}}\left|h_{i d}(k)\right|^{2}<\Lambda-\left|h_{s d}(k)\right|^{2}\right]=0$, $\lim _{\gamma_{s} \rightarrow \infty} \operatorname{Pr}\left[\left|h_{s i}(k)\right|^{2}-\left|h_{p i}(k)\right|^{2} \gamma_{p} \Lambda>\Lambda\right]=\frac{1}{\rho_{i}^{-1} \lambda_{s d-p d}^{-1} v+1}$, and $\lim _{\gamma_{s} \rightarrow \infty} \operatorname{Pr}\left[\left|h_{s j}(k)\right|^{2}-\left|h_{p j}(k)\right|^{2} \gamma_{p} \Lambda<\Lambda\right]=\frac{\alpha}{\lambda_{s d-p d}^{-1} v+\rho_{j}} \cdot \frac{2^{2 R}-1}{\lambda_{s d-p d}}$, where $\alpha=\gamma_{p} / \gamma_{s}, v=\alpha\left(2^{2 R}-1\right)$ and $\rho_{i}=\lambda_{s i-p i} / \lambda_{s d-p d}$. Combining these results and (C.4), we obtain

$$
\begin{aligned}
\lim _{s} \rightarrow \infty & \operatorname{Pr}\left[I_{\text {best-relay }}(k)<R, D=D_{m} \mid \hat{H}_{s}(k)=H_{0}\right] \\
= & \pi_{2} \frac{\alpha^{\left\|D_{m}\right\|+1} v\left\|D_{m}\right\| !}{\left(\lambda_{s d-p d}^{-1} v+1\right) \prod_{i \in D_{m}} \delta_{i}} \prod_{i \in D_{m}} \frac{1}{\rho_{i}^{-1} \lambda_{s d-p d}^{-1} v+1} \\
& \times \prod_{j \in \bar{D}_{m}} \frac{\alpha}{\lambda_{s d-p d}^{-1} v+\rho_{j}} \cdot\left(\frac{2^{2 R}-1}{\lambda_{s d-p d}}\right)^{M+1}
\end{aligned}
$$

Besides, term $\lim _{\gamma_{s} \rightarrow \infty} \operatorname{Pr}\left[I_{\text {direct }}(k)<R, D=\emptyset \mid \hat{H}_{s}(k)=H_{0}\right]$ is easily found as

$$
\begin{aligned}
& \lim _{\gamma_{s} \rightarrow \infty} \operatorname{Pr}\left[I_{\text {direct }}(k)<R, D=\emptyset \mid \hat{H}_{s}(k)=H_{0}\right] \\
& =\frac{\pi_{2} \alpha^{M+1}}{\lambda_{s d-p d}^{-1} \nu+1} \prod_{i=1}^{M} \frac{1}{\lambda_{s d-p d}^{-1} v+\rho_{i}} \cdot \frac{\left(2^{R}-1\right)\left(2^{2 R}-1\right)^{M}}{\left(\lambda_{s d-p d}\right)^{M+1}}
\end{aligned}
$$

where $\nu=\alpha\left(2^{R}-1\right)$. Hence, substituting (C.5), (C.6) and (23) into (C.1) yields

$$
d_{\text {non-ACK,multiple }}+2(M+1) r=M+1
$$

which completes DMT analysis of the non-ACK based bestrelay cooperation scheme.

\section{B. Lemma 4 (ACK Based Selective Best-Relay Cooperation Scheme)}

This lemma shows a DMT for the ACK based selective bestrelay cooperation scheme. Similar to (C.1), the diversity gain of the ACK based selective best-relay cooperation scheme is defined as

$$
d_{\mathrm{ACK}, \text { multiple }}=-\lim _{\lambda_{s d-p d} \rightarrow \infty} \frac{\log \left(\text { Pout }_{\text {ACK,multiple }}^{\text {floor }}\right)}{\log \left(\lambda_{s d-p d}\right)}
$$




$$
\begin{aligned}
& \psi_{S_{m}(n)}=\frac{\exp \left(-\sum_{i \in S_{m}(n)} \frac{\Lambda}{\sigma_{i d}^{2}}\right)}{\left(1+\sum_{i \in S_{m}(n)} \frac{\sigma_{p d}^{2} \gamma_{p} \Lambda}{\sigma_{i d}^{2}}\right)\left(1-\sum_{i \in S_{m}(n)} \frac{\sigma_{s d}^{2}}{\sigma_{i d}^{2}}\right)} \\
& +\left[\frac{1}{1+\frac{\sigma_{s d}^{2}}{\sigma_{p d}^{2} \gamma_{p} \Delta}+\sum_{i \in S_{m}(n)} \frac{\sigma_{s d}^{2}(\Lambda-\Delta)}{\sigma_{i d}^{2} \Delta}}-\frac{1}{1-\sum_{i \in S_{m}(n)} \frac{\sigma_{s d}^{2}}{\sigma_{i d}^{2}}}\right] \frac{\exp \left(-\frac{\Delta}{\sigma_{s d}^{2}}-\sum_{i \in S_{m}(n)} \frac{\Lambda-\Delta}{\sigma_{i d}^{2}}\right)}{\left(1+\sum_{i \in S_{m}(n)} \frac{\sigma_{p d}^{2} \gamma_{p} \Lambda}{\sigma_{i d}^{2}}\right)} \\
& \text { Pout }_{\text {ACK, multiple }}^{\text {floor }}=\sum_{m=1}^{2^{M}-1} \lim _{\gamma_{s} \rightarrow \infty} \operatorname{Pr}\left[I_{\text {best-relay }}(k)<R, D=D_{m}, I_{\text {direct }}(k)<R \mid \hat{H}_{s}(k)=H_{0}\right] \\
& +\lim _{\gamma_{s} \rightarrow \infty} \operatorname{Pr}\left[I_{\text {direct }}(k)<R, D=\emptyset \mid \hat{H}_{s}(k)=H_{0}\right] \\
& \lim _{\gamma_{s} \rightarrow \infty} \operatorname{Pr}\left\{\max _{i \in D_{m}} x_{i}<-y+z v, 0<-y+z \nu\right\} \\
& =\iint_{0<-y+z \nu} \frac{1}{\sigma_{s d}^{2} \sigma_{p d}^{2}} \exp \left(-\frac{y}{\sigma_{s d}^{2}}-\frac{z}{\sigma_{p d}^{2}}\right) \prod_{i \in D_{m}}\left[1-\exp \left(-\frac{-y+z v}{\sigma_{i d}^{2}}\right)\right] d y d z \\
& =\iint_{0<-y+z \nu} \frac{1}{\sigma_{s d}^{2} \sigma_{p d}^{2}} \exp \left(-\frac{y}{\sigma_{s d}^{2}}-\frac{z}{\sigma_{p d}^{2}}\right) \prod_{i \in D_{m}} \frac{-y+z v}{\sigma_{i d}^{2}} d y d z \\
& \leq \frac{v^{\left\|D_{m}\right\|}}{\prod_{i \in D_{m}} \sigma_{i d}^{2}} \iint_{0<-y+z \nu} \frac{z^{\left\|D_{m}\right\|}}{\sigma_{s d}^{2} \sigma_{p d}^{2}} \exp \left(-\frac{y}{\sigma_{s d}^{2}}-\frac{z}{\sigma_{p d}^{2}}\right) d y d z \\
& =\frac{v^{\left\|D_{m}\right\|}}{\prod_{i \in D_{m}} \sigma_{i d}^{2}} \int_{0}^{\infty} \frac{1}{\sigma_{s d}^{2}} \exp \left(-\frac{y}{\sigma_{s d}^{2}}\right) d y \int_{\frac{y}{\nu}}^{\infty} \frac{z^{\left\|D_{m}\right\|}}{\sigma_{p d}^{2}} \exp \left(-\frac{z}{\sigma_{p d}^{2}}\right) d z
\end{aligned}
$$

where Pout $\mathrm{ACK}_{\mathrm{AC} \text {,multiple }}^{\text {flor }}$ is an outage probability floor of the ACK based selective best-relay cooperation scheme. Denoting $\gamma_{p}=\alpha \gamma_{s}$ and letting $\gamma_{s} \rightarrow+\infty$, we can obtain an outage probability floor of the ACK based selective cooperation scheme from (37) as Eq. (C.9) at the top of the following page. Letting $x_{i}=\left|h_{i d}\right|^{2}, y=\left|h_{s d}\right|^{2}$ and $z=\left|h_{p d}\right|^{2}$, we rewrite $\lim _{\gamma_{s} \rightarrow \infty} \operatorname{Pr}\left\{\max _{i \in D_{m}}\left|h_{i d}\right|^{2}<\Lambda-\left|h_{s d}\right|^{2}+\left|h_{p d}\right|^{2} \gamma_{p} \Lambda,\left|h_{s d}\right|^{2}<\right.$ $\left.\Delta+\left|h_{p d}\right|^{2} \gamma_{p} \Delta\right\}$ as $\lim _{\gamma_{s} \rightarrow \infty} \operatorname{Pr}\left\{\max _{i \in D_{m}} x_{i}<\Lambda-y+z \gamma_{p} \Lambda, 0<\right.$ $\left.\Delta-y+z \gamma_{p} \Delta\right\}$, where $\Lambda=\left(2^{2 R}-1\right) / \gamma_{s}$ and $\Delta=$ $\left(2^{R}-1\right) / \gamma_{s}$. By denoting $\alpha=\gamma_{p} / \gamma_{s}, v=\alpha\left(2^{2 R}-1\right)$ and $\nu=\alpha\left(2^{R}-1\right)$, the above term is further simplified to $\lim _{\gamma_{s} \rightarrow \infty} \operatorname{Pr}\left\{\max _{i \in D} x_{i}<-y+z v, 0<-y+z \nu\right\}$, which is calculated as Eq. (C.10), where the second equation is resulted from Theorem 1 and the second integral inside the last equation, i.e, $\int_{\frac{y}{\nu}}^{\infty} \frac{z^{\left\|D_{m}\right\|}}{\sigma_{p d}^{2}} \exp \left(-\frac{z}{\sigma_{p d}^{2}}\right) d z$, is given by

$$
\begin{aligned}
& \int_{\frac{y}{\nu}}^{\infty} \frac{z^{\left\|D_{m}\right\|}}{\sigma_{p d}^{2}} \exp \left(-\frac{z}{\sigma_{p d}^{2}}\right) d z \\
& =\sum_{r=0}^{\left\|D_{m}\right\|} \frac{\left\|D_{m}\right\| ! \sigma_{p d}^{2\left(\left\|D_{m}\right\|-r\right)}}{r !}\left(\frac{y}{\nu}\right)^{r} \exp \left(-\frac{y}{\nu \sigma_{p d}^{2}}\right)
\end{aligned}
$$

Substituting (C.11) into (C.10) and denoting $\delta_{i}=$ $\lambda_{i d-p d} / \lambda_{s d-p d}$, we can obtain

$$
\begin{aligned}
& \lim _{\gamma_{s} \rightarrow \infty} \operatorname{Pr}\left\{\max _{i \in D_{m}} x_{i}<-y+z v, 0<-y+z \nu\right\} \\
& \leq \sum_{r=0}^{\left\|D_{m}\right\|} \frac{\left\|D_{m}\right\| ! \alpha^{\left\|D_{m}\right\|+1}}{\left(\nu \lambda_{s d-p d}^{-1}+1\right)^{r+1} \prod_{i \in D_{m}} \delta_{i}} \cdot \frac{\left(2^{2 R}-1\right)^{\left\|D_{m}\right\|}\left(2^{R}-1\right)}{\left(\lambda_{s d-p d}\right)^{\left\|D_{m}\right\|+1}}
\end{aligned}
$$

Using (C.12), we obtain

$$
\begin{aligned}
& \lim _{\gamma_{s} \rightarrow \infty} \operatorname{Pr}\left[I_{\text {best-relay }}(k)<R, D=D_{m}, I_{\text {direct }}(k)<R \mid \hat{H}_{s}(k)=H_{0}\right] \\
& \leq \sum_{r=0}^{\left\|D_{m}\right\|} \frac{\left\|D_{m}\right\| ! \pi_{2} \alpha^{M+1}}{\left(\nu \lambda_{s d-p d}^{-1}+1\right)^{r+1} \prod_{i \in D_{m}} \delta_{i}} \prod_{i \in D_{m}} \frac{1}{\rho_{i}^{-1} \lambda_{s d-p d}^{-1} v+1} \\
& \quad \times \prod_{j \in \bar{D}_{m}} \frac{1}{\lambda_{s d-p d}^{-1} v+\rho_{j}} \cdot \frac{\left(2^{2 R}-1\right)^{M}\left(2^{R}-1\right)}{\left(\lambda_{s d-p d}\right)^{M+1}}
\end{aligned}
$$

Meanwhile, the second term in the right-hand side of (C.9) is found as (C.6). Hence, substituting (C.6), (C.13) and (23) into (C.8) yields

$$
d_{\mathrm{ACK}, \text { multiple }} \geq M+1-(2 M+1) r
$$

Besides, an outage probability floor of the ACK based selective best-relay cooperation scheme can be described as 


$$
\begin{aligned}
\operatorname{Pout}_{\mathrm{ACK}, \text { multiple }}^{\text {floor }} \geq & \lim _{\gamma_{s} \rightarrow \infty} \operatorname{Pr}\left[I_{\text {direct }}(k)<R, D=\emptyset \mid \hat{H}_{s}(k)=H_{0}\right] \\
= & \frac{\pi_{2} \alpha^{M+1}}{\lambda_{s d-p d}^{-1} \nu+1} \prod_{i=1}^{M} \frac{1}{\lambda_{s d-p d}^{-1} v+\rho_{i}} \\
& \times \frac{\left(2^{R}-1\right)\left(2^{2 R}-1\right)^{M}}{\left(\lambda_{s d-p d}\right)^{M+1}}
\end{aligned}
$$

Using (C.15), we can obtain a DMT of the ACK based selective best-relay cooperation scheme as given by

$$
d_{\mathrm{ACK}, \text { multiple }} \leq M+1-(2 M+1) r
$$

Therefore, according to the squeeze theorem, the DMT of the ACK base selective best-relay cooperation scheme is obtained from (C.14) and (C.16) as

$$
d_{\mathrm{ACK}, \text { multiple }}=M+1-(2 M+1) r
$$

Now, we complete the DMT analysis for the ACK based selective best-relay cooperation scheme.

\section{REFERENCES}

[1] J. Mitola and G. Q. Maguire, "Cognitive radio: making software radios more personal," IEEE Personal Commun., vol. 6, pp. 13-18, 1999.

[2] Y. Zou, Y.-D. Yao, and B. Zheng, "Cooperative relay techniques for cognitive radio systems: spectrum sensing and secondary user transmissions," IEEE Commun. Mag., vol. 50, no. 4, Apr. 2012.

[3] IEEE 802.22 Working Group, "IEEE P802.22/D1.0 draft standard for wireless regional area networks part 22: cognitive wireless RAN medium access control (MAC) and physical layer (PHY) specifications: policies and procedures for operation in the TV bands," Apr. 2008.

[4] Y. Zou, Y.-D. Yao, and B. Zheng, "Outage probability analysis of cognitive transmissions: impact of spectrum sensing overhead," IEEE Trans. Wireless Commun., vol. 9, no. 8, pp. 2676-2688, Aug. 2010.

[5] Y. Zou, Y.-D. Yao, and B. Zheng, "Cognitive transmissions with multiple relays in cognitive radio networks," IEEE Trans. Wireless Commun., vol. 10, no. 2, pp. 648-659, Feb. 2011.

[6] J. N. Laneman, D. N. C. Tse, and G. W. Wornell, "Cooperative diversity in wireless networks: efficient protocols and outage behavior," IEEE Trans. Inf. Theory, vol. 50, no. 12, pp. 3062-3080, 2004.

[7] J. N. Laneman and G. W. Wornell, "Distributed space-time-coded protocols for exploiting cooperative diversity in wireless networks," IEEE Trans. Inf. Theory, vol. 49, no. 10, pp. 2415-2425, Oct. 2003.

[8] Y. Zou, B. Zheng, and J. Zhu, "Outage analysis of opportunistic cooperation over Rayleigh fading channels," IEEE Trans. Wireless Commun., vol. 8, no. 6, pp. 3077-3085, June 2009.

[9] A. Bletsas, H. Shin, M. Z. Win, and A. Lippman, "A simple cooperative diversity method based on network path selection," IEEE J. Sel. Areas Commun., vol. 24, no. 3, pp. 659-672, Mar. 2006.

[10] E. Beres and R. S. Adve, "Selection cooperation in multi-source cooperative networks," IEEE Trans. Wireless Commun., vol. 7, no. 1, pp. 118-127, Jan. 2008

[11] A. Ghasemi and E. S. Sousa, "Collaborative spectrum sensing for opportunistic access in fading environment," in Proc. 2005 IEEE DySPAN.

[12] G. Ganesan and Y. G. Li, "Cooperative spectrum sensing in cognitive radio-part I: two user networks," IEEE Trans. Wireless Commun., vol. 6, no. 6, pp. 2204-2213, 2007.

[13] K. Lee and A. Yener, "Throughput enhancing cooperative spectrum sensing strategies for cognitive radios," in Proc. 2007 Asilomar Conf. Signals, Syst. Comput.

[14] W. Saad, Z. Han, M. Debbah, A. Hjorungnes, and T. Basar, "Coalitional games for distributed collaborative spectrum sensing in cognitive radio networks," in Proc. 2009 IEEE INFOCOM.
[15] J. Ma, G. Zhao, and Y. Li, "Soft combination and detection for cooperative spectrum sensing in cognitive radio networks," IEEE Trans. Wireless Commun., vol. 7, no. 11, pp. 4502-4507, Nov. 2008.

[16] Y. Zou, Y.-D. Yao, and B. Zheng, "A selective-relay based cooperative spectrum sensing scheme without dedicated reporting channels in cognitive radio networks," IEEE Trans. Wireless Commun., vol. 10, no. 4, Apr. 2011.

[17] Y. Zou, J. Zhu, B. Zheng, and Y.-D. Yao, "An adaptive cooperation diversity scheme with best-relay selection in cognitive radio networks," IEEE Trans. Signal Process., vol. 58, no. 10, pp. 5438-5445, Oct. 2010.

[18] L. Zheng and D. Tse, "Diversity and multiplexing: a fundamental tradeoff in multiple antenna channels," IEEE Trans. Inf. Theory, vol. 49, no. 5, pp. 1073-1096, May 2003.

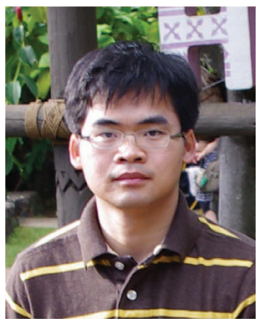

Yulong Zou received his B.Eng. degree in Information Engineering from Nanjing University of Posts and Telecommunications (NUPT), Nanjing China, in 2006. He is currently working toward his dual Ph.D. degree at the Institute of Signal Processing and Transmission of NUPT, Nanjing China, and the Electrical and Computer Engineering Department of Stevens Institute of Technology (SIT), NJ USA. His research interests span a wide range of topics in wireless communications and signal processing, including cooperative communications, space-time coding, network coding, and cognitive radio. Recently, he has been working on the cooperative relay techniques in cognitive radio networks, opportunistic distributed space-time coding in cooperative wireless networks, and fulldiversity high-rate network coding for cellular systems (e.g., LTE/IMTadvanced and beyond).

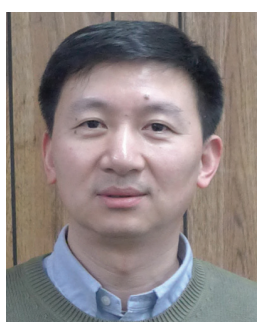

Yu-Dong Yao (S'88-M'88-SM'94-F'11) has been with Stevens Institute of Technology, Hoboken, New Jersey, since 2000 and is currently a professor and department director of electrical and computer engineering. He is also a director of Stevens' Wireless Information Systems Engineering Laboratory (WISELAB). Previously, from 1989 and 1990, he was at Carleton University, Ottawa, Canada, as a Research Associate working on mobile radio communications. From 1990 to 1994, he was with Spar Aerospace Ltd., Montreal, Canada, where he was involved in research on satellite communications. From 1994 to 2000, he was with Qualcomm Inc., San Diego, CA, where he participated in research and development in wireless code-division multiple-access (CDMA) systems.

He holds one Chinese patent and twelve U.S. patents. His research interests include wireless communications and networks, spread spectrum and CDMA, antenna arrays and beamforming, cognitive and software defined radio (CSDR), and digital signal processing for wireless systems. Dr. Yao was an Associate Editor of IEEE COMMUNICATIONS LETTERS and IEEE TRANSACTIONS ON VEHICULAR TECHNOLOGY, and an Editor for IEEE TRANSACTIONS ON WIRELESS COMMUNICATIONS. He received the B.Eng. and M.Eng. degrees from Nanjing University of Posts and Telecommunications, Nanjing, China, in 1982 and 1985, respectively, and the Ph.D. degree from Southeast University, Nanjing, China, in 1988, all in electrical engineering.

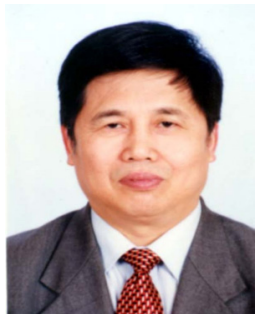

Baoyu Zheng received B.S. and M.S. degrees from the Department of Circuit and Signal System, NUPT, in 1969 and 1981, respectively. Since then, he has been engaged in teaching and researching at Signal and Information Processing. He is a full professor and doctoral advisor at NUPT. His research interests span the broad area of the intelligent signal processing, wireless network and signal processing for modern communication, and the quantum signal processing. 